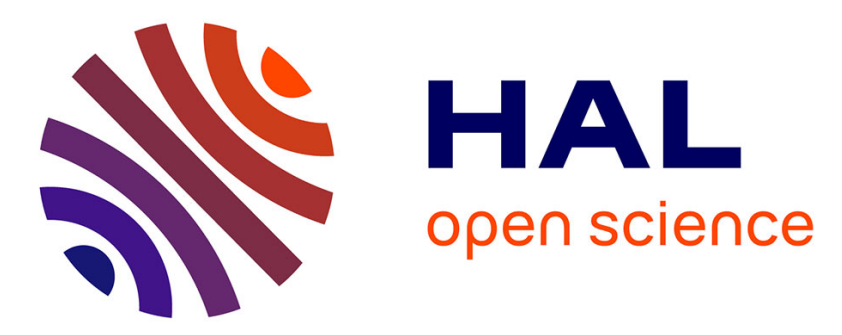

\title{
Enhanced pseudo-piezoelectric dynamic force sensors based on inkjet-printed electrostrictive terpolymer
} Qing Liu, Minh-Quyen Le, Pierre-Jean Cottinet, Jean-Fabien Capsal, C. Richard

\section{- To cite this version:}

Qing Liu, Minh-Quyen Le, Pierre-Jean Cottinet, Jean-Fabien Capsal, C. Richard. Enhanced pseudopiezoelectric dynamic force sensors based on inkjet-printed electrostrictive terpolymer. Organic Electronics, 2019, 67, pp.259-271. 10.1016/j.orgel.2019.01.028 . hal-02165410

\author{
HAL Id: hal-02165410 \\ https://hal.science/hal-02165410
}

Submitted on 22 Oct 2021

HAL is a multi-disciplinary open access archive for the deposit and dissemination of scientific research documents, whether they are published or not. The documents may come from teaching and research institutions in France or abroad, or from public or private research centers.
L'archive ouverte pluridisciplinaire HAL, est destinée au dépôt et à la diffusion de documents scientifiques de niveau recherche, publiés ou non, émanant des établissements d'enseignement et de recherche français ou étrangers, des laboratoires publics ou privés.

\section{다)(1) $(5$}

Distributed under a Creative Commons Attribution - NonCommerciall 4.0 International 


\title{
Enhanced Pseudo-piezoelectric Dynamic Force Sensors based on Inkjet-printed Electrostrictive Terpolymer
}

\author{
Qing Liu ${ }^{a}$, Minh Quyen Le ${ }^{a}$, Claude Richard ${ }^{a}$, Richard Liang ${ }^{b}$, Pierre-Jean Cottinet ${ }^{a}$, Jean-Fabien Capsal ${ }^{* a}$ \\ ${ }^{a}$ Université de Lyon, INSA-Lyon, LGEF, EA682, F-69621, Villeurbanne, France \\ ${ }^{b}$ High-Performance Materials Institute, FAMU-FSU College of Engineering, Florida State University 2005 Levy Ave, \\ Tallahassee, FL 32310, United States \\ *Correspondence to: J.-F. Capsal (E-mail: jean-fabien.capsal@insa-lyon.fr)
}

\begin{abstract}
Printed electromechanical devices are an alternative approach for the fabrication of integrated force sensor networks on large-area flexible polymer foils. In the present contribution, we report on a novel parallelplates capacitive force sensor device based on an electrostrictive poly(vinylidenefluoride-trifluoroethylenechlorotrifluoroethylene) (P(VDF-TrFE-CTFE)) terpolymer that exhibited the best electromechanical coupling effect through the drop-on-demand (DoD) inkjet printing technology. Since the ejection of a printable ink droplet is one of the most essential steps for inkjet printing, the adaptable printing parameters depending on the ink delivery system as well as ink formula rheology were first investigated. Successfully printed patterns rely on a controlled drying process of the inks deposited on the flexible polyimide foil, which was also discussed. The substrate temperature was increased for achieving homogeneous polymer patterns. Such a printing technique eventually made it possible to obtain flawless polymer thin layers of several microns, preventing the devices from the risk of electrical failure due to short-circuiting. The capacitive devices were evaluated by proposition of a dynamic force sensor. Sensor devices based on an electrostrictive terpolymer were induced to yield significant polarization thanks to a bias voltage whereas a sensor device of poly(vinylidenefluoride-trifluoroethylene) $\mathrm{P}(\mathrm{VDF}-\mathrm{TrFE})$ copolymer must be poled under a high electric field with a very high risk of breakdown failure. The pseudo-piezoelectric coefficient $d_{33 e q}$ was a strict function of the applied electric field and reached as high as $81.6 \mathrm{pC} \mathrm{N}^{-1}$ under $33.33 \mathrm{~V} / \mu \mathrm{m}$. The fabrication process in the present study holds high potential in large-area printed terpolymer sensor networks without a poling process as each sensor corresponds to a pixel of the sensing array.
\end{abstract}

Keywords: Inkjet printing, Electroactive polymer, Pseudo-piezoelectric, Force sensor.

\section{Introduction}

A sensor is defined as a device that is capable of responding to certain kinds of physical input excitations and converting them into electrical output signals, compatible with signal processing circuits which ensure the signal readability. In particular, force sensor elements switch the mechanical deformation 
into readable electrical output signals with conformal characteristics, including excitation frequency, phase and amplitude, etc. Accurately gauging and monitoring the small- and large-scale force stimuli, occasionally in-situ, could benefit the numerous potentials in fields of smart packaging [1], biological micromanipulation [2], acoustic transducers [3] and medical devices [4], etc. Meanwhile, challenges have also been taken on, aimed at determining the specific design and a simple fabrication of the sensor devices. For example, embedded sensors in industrial microelectronic devices and flying objects are preferentially miniaturized and compact but durable and flexible, while sensors for atomic force sensing and microscale end-use demand ultrahigh sensitivities and accuracy. Thanks to the ever-growing development of printing technologies, sensor fabrication based on large-area printed electronic devices is booming. Conventional approaches for materials distribution consist in applying the sensing material layers with a solutionprocessing ink transfer technique via a contact mode such as roll-to-roll gravure printing, lithography, micro-contact printing, and so on [5-7].

Soft/rigid rolling rods, fabricated by the micromachining process and carrying the relief pattern information, are able to deliver and distribute the inks on the large-area flexible substrates. Nevertheless, it is very challenging to obtain a high quality of sensing material patterns with the afore-mentioned printing techniques. They rely on several quality controls in each step involving the ink delivery system, the surface characteristics of the flexible substrate and ink formula rheology, etc. The inkjet printing technology is an emerging and noncontact ink distribution method, potentially rendering it possible to overcome these drawbacks, especially those related to the low printing qualities and resolutions observed in contact printing. Moreover, this direct patterning technique also endues possible the deposition of drop-on-demand materials, enabling the fabrication of miniaturized objects with a more delicate structure. This technique works only with a liquid ink solution, firstly transported and stored in the micro-chamber. Ink solutions are ejected from the printing nozzle, often as a sequence of droplets, by thermal or electromechanical elements to a precisely controlled location. The droplets then detach from the nozzle orifice and hit the substrate in a stable trajectory. A successful inkjet printed pattern is the result of compatibility between the ink rheology, the drop ejection system and a wettable substrate.

Inkjet printing has the advantage of a highly precise ink allocation and standard droplet volumes down to the picoliter scale facilitating the miniaturization of the printed objects, in the case of for instance embedded sensors, which in turn reduce excessive power consumption in mobile applications (e.g., smartphones, tablets, healthcare monitors). Furthermore, inkjet printing is intrinsically combined with the numerous merits of the classic printing techniques, e.g., a high throughput and standardization [8-10]. Additionally, this technique is highlighted as being cost-effective, step-saving and leading to the low waste of material, making it quite attractive for printed microelectronic sensors and actuators [11, 12].

The fluorinated $\mathrm{P}(\mathrm{VDF}-\mathrm{TrFE}-\mathrm{CTFE})$ terpolymer is known to possess a superior electromechanical coupling effect due to its excellent electrostrictive behavior. It is thus able to realize the conversion between mechanical excitation and electric signals[13, 14]. However, the major drawback of the terpolymer is the inevitable requirement of a high electric field, limiting its application range. This technical dilemma can be tackled either by enhancing the dielectric permittivity or miniaturizing the sensing elements. Over the 
decades, the dielectric performance of electroactive materials has been hugely improved by introducing conductive metal-ligand pairs[15, 16], carbon homologues[17] and ceramic relaxor materials[18] into the polymer matrix. Even though the compatibility issue has been eliminated through chemistry, there are still some rather severe disadvantages, such as reduced dielectric strength and unwanted increase in mechanical strength. An alternative method to tackling the problems pragmatically in case of high electric fields is to miniaturize the geometric parameters, for example, the thickness of parallel-plate capacitive sensors and actuator devices. The drop-on-demand inkjet printing technique has thus been carefully rated and selected based on the materials distribution method as it shows numerous advantages compared to other printing technologies. On top of that, a precisely controlled thickness of the printed sensing layer leads to high sensitivity with a low power supply.

This study reports on a thickness-controlled parallel-plate capacitive-like sensor using a flexible polyimide substrate. The inkjet printing protocol was optimized prior to the manufacturing of the patterned ferroelectric and electrostrictive sensing polymer layers on the flexible polyimide foils. The characteristics of morphology and the dielectric features of printed terpolymer layers have been explored, following by a validation of the proposed printed electroactive polymer based on two types of dynamic impact force sensors. The performances in terms of sensitivity and reliability for these both sensors have been eventually evaluated.

\section{Theoretical model of electrostrictive polymer}

Since a sensor is a converter capable of transforming nonelectric energy into an electric signal, multiple transformation or change steps are required prior to the output of an electric signal, especially with a force sensor based on the electromechanical coupling mechanism of a-usually dielectric-material. The effect that has been revealed is a common feature in ferroelectric and electrostrictive polymers [19-21]. Their geometric dimensions change in response to an external physical excitation, such as an electric field, or a pulse of a dynamic force can generate a variation of the electrical displacement due to a combined underlying mechanism of piezoelectric, electrostrictive and electrostatic effects. For a pure ferroelectric polymer in the thickness direction, the tensor interactions between mechanical stress, strain, electric field and displacement can be expressed in the following constitutive equations[22, 23]:

$$
\begin{aligned}
& S_{33}=s_{33}^{E} T_{33}+d_{33} E_{33} \\
& D_{33}=d_{33} T_{33}+\varepsilon_{33}^{T} E_{33}
\end{aligned}
$$

where $S_{33}$ is the mechanical strain out-of-plane of the polymer unimorph; $s_{33}^{E}$ is the elastic compliance; $T_{33}$ is the mechanical stress along the thickness direction; $d_{33}$ is the piezoelectric coefficient; $E_{33}$ is the electric field along the thickness direction; $\varepsilon_{33}^{T}$ is the dielectric constant under $E_{33}$; and $D_{33}$ is the electric displacement. Under an out-of-plane electric field $E$, the dipoles aligning with the electric excitation give rise to a large polarization within the ferroelectric polymer, and thus a huge remnant polarization $P_{r}$ even 
though the electric excitation is once switched off, as illustrated in Figure 1. Likewise, when a dynamic force is applied to a capacitor-like ferroelectric device, the geometric change causes a variation in its capacitance, therefore accompanied with a varying electrical polarization $\Delta P$. Such variations give rise to an electric leakage current to be read by a supplementary outside circuit, making it possible to gauge the applied dynamic force by only measuring the induced electric current output. However, relaxor ferroelectric materials or electrostrictive matter present a very slim $D-E$ hysteresis and thus a barely detectable remnant polarization $P_{r}$ (schema in Figure 1b) [13, 14]. Since the electrostrictive effect dominates the origins of thickness contraction of the terpolymer in response to the applied electric field, equations 1 and 2 can be rewritten as linearized constitutive expressions [24, 25]:

$$
\begin{aligned}
& S_{33}=s_{33}^{E} T_{33}+M_{33} E_{33}^{2} \\
& D_{3}=2 M_{33} E_{33} T_{33}+\varepsilon_{33}^{T} E_{33}
\end{aligned}
$$

Here, $M_{33}$ is the global electrostrictive coefficient across the thickness direction. The cluster $2 M_{33} E_{33}$ equivalent to the $d_{33}$ is the so-called pseudo-piezoelectric coefficient under an electric field. A large polarization can also be induced with the help of a bias voltage for the non-piezoelectric polymers in our case. In a similar mechanism to ferroelectrics, the thickness deformation yields a change in the electric displacement with a short-circuit leakage current $I[26,27]$ :

$$
I=A \cdot \frac{\partial D_{33}}{\partial t}
$$

where $A$ is the electrode area covering the electrostrictive terpolymer and $\frac{\partial D_{33}}{\partial t}$ is the electrical displacement variation during a defined time. Equation 5 can be rewritten by including equation 4 in the $\frac{\partial D_{33}}{\partial t}$ cluster:

$$
I=A\left[\frac{\partial E_{33}}{\partial t}\left(\varepsilon_{33}^{T}+\frac{2 M_{33} S_{33}-6 M_{33}^{2} E_{33}^{2}}{s_{33}^{E}}\right)+\frac{2 M_{33} \frac{\partial S_{33}}{\partial t} E_{33}}{s_{33}^{E}}\right]
$$

Herein, a DC bias voltage was applied in the thickness direction of the electrostrictive terpolymers. The electric field variation rate $\frac{\partial E_{33}}{\partial t}$ was significantly limited as the thickness change was maintained in the restrictive scope. Hence, the short circuit current $I$ can be simplified as [28-30]:

$$
I=A \cdot\left[2 M_{33} E_{3} Y \frac{\partial S_{33}}{\partial t}\right]
$$

$I$ is intrinsically regulated by the pseudo-piezoelectric coefficient $2 M_{33} E_{3}$ of the electrostrictive terpolymer and the time derivative of the strain $\frac{\partial S_{33}}{\partial t}$ across the thickness direction. 

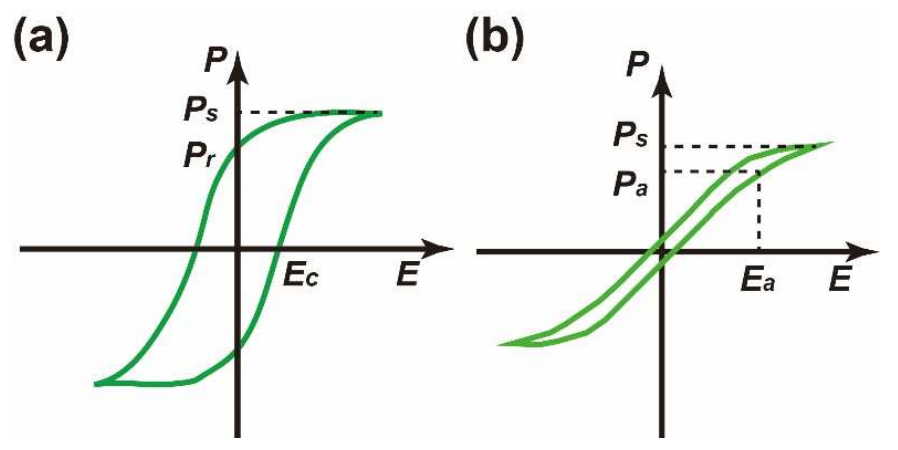

Figure 1 Schemata of polarization (P)-electric field (E) hysteresis for a typical ferroelectric copolymer (P(VDF-TrFE)) (a) and an electrostrictive terpolymer (P(VDF-TrFE-CTFE)) (b). The ferroelectric copolymer exhibited wide hysteresis loops with a significant remnant polarization $\left(P_{r}\right)$, whereas its electrostrictive counterpart presented a narrow $P$ - $E$ hysteresis with a trace of remnant dipole polarization.

As motivated by using the theoretical model, the ferroelectric P(VDF-TrFE) copolymer and electrostrictive $\mathrm{P}(\mathrm{VDF}-\mathrm{TrFE}-\mathrm{CTFE})$ terpolymer were employed as sensing materials on a parallel-plate capacitor-like impact force sensor thanks to its high dielectric permittivity, low loss and excellent (pseudo)piezoelectric coefficient which expectedly made it possible to achieve a superior electromechanical coupling phenomenon. In addition, a less rigid and low Young's modulus would benefit the large geometric deformation and strain-changing rate, in favor of an electric response to the dynamic mechanical excitation.

\section{Materials and Methods}

\section{1 Selection of Materials}

Commercially available passive and thermoresistant 50- $\mu \mathrm{m}$ thick polyimide (PI) foils (Dupont ${ }^{\mathrm{TM}}$ Kapton ${ }^{\circledR}$ HN, USA) served as the flexible substrate for the terpolymer dynamic force sensors. Prior to the ink printing, Kapton films were successively rinsed with acetone, ethanol and isopropanol, to ensure that the reproducible and wettable surface of the flexible foils was free from contaminants. Afterwards, in order to endue an excellent adhesion between the Kapton substrate and the inert metal electrode as well as the solid sensing polymer layers, fresh Kapton films were subjected to UV-Ozone exposure whereby the surface energy of the PI substrate was greatly improved. This way, the surface tension of the PI substrate matched the surface properties of those non-parasitic elements.

The sensing polymer layer, the $\mathrm{P}(\mathrm{VDF}-\mathrm{TrFE})$ copolymer with a molar ratio of 55:45/VDF:TrFE and the P(VDF-TrFE-CTFE) terpolymer with a molar ratio of 61.0:30.1:8.9/VDF:TrFE:CTFE, were both purchased from Piezotech S.A.S (Arkema, France) and used as received. The number-average molecular weight characterization were approximately to be $450,000 \mathrm{~g} \mathrm{~mol}^{-1}$ for copolymer, 500,000 $\mathrm{g} \mathrm{mol}^{-1}$ for terpolymer indicated by the material producer (cf. Piezotech) [31]. The solvents employed in the following substrate pretreatment and ink formulation were all obtained from Sigma-Aldrich and used as received unless otherwise specified. Both kinds of P(VDF-TrFE) and P(VDF-TrFE-CTFE) ink formulation at a 
concentration of $1 \mathrm{wt} \%$ were prepared by dissolving the both polymer granules in triethyl phosphate (TEP) solvent.

\section{2 Device Fabrication based on Inkjet Printing}

Freshly rinsed PI foil films were firstly introduced into a conventional oven for $20 \mathrm{~min}$ at $150^{\circ} \mathrm{C}$. After heat equilibrium, the PI foils were subjected to UV-Ozone exposure, thereby improving the ink wettability and adhesion with the printed components. The circular bottom Au electrode was then sputtered using a customized shadow mask with the hole area of $78.54 \mathrm{~mm}^{2}$. The thickness of the deposited electrode was able to be precisely controlled via modulating the time and DC current of Au exposure and thus was estimated at a thickness of about $40 \mathrm{~nm}$. Prior to filling the inks to print, the solution was filtered through a Teflon filter with $1 \mu \mathrm{m}$ custom-sized pores. The circular sensing polymer layer, with a diameter of $15 \mathrm{~mm}$, was successively deposited on the bottom Au electrode in rather good alignment using a Dimatrix cartridge (SE-128 AA) with a specified droplet volume of 50 picoliter mounted on a commercial PiXDRO LP50 inkjet printer (Meyer Burger Technologies, Switzerland).

Meanwhile, the PI substrate was continuously heated at $90{ }^{\circ} \mathrm{C}$ to favor the vaporization dynamics of the solvent that had a relatively high boiling point. Concretely, the printing protocol was chosen to deposit the uniform and homogeneous polymer layers using jettable droplets relying on the preferential printing parameters, with a printable morphology, a falling velocity of $1 \mathrm{~m} \mathrm{~s}^{-1}$ and a firing frequency of $1 \mathrm{kHz}$ under an optimal equilibrium ink pressure of -21.7 mbar. The print head was powered by a voltage of $120 \mathrm{~V}$, with a pulse width of $6 \mu \mathrm{s}$ for the raising step, $8 \mu \mathrm{s}$ for the dwelling step and $4 \mu \mathrm{s}$ for the falling step in the injet printing protocol. In order to obtain the desired sensing layer with the predetermined thickness of approximately $10 \mu \mathrm{m}$, multiple repetitions of printing were manipulated. The both freshly printed polymer layers were dried over several minutes, after which they were moved into a conventional oven and subjected to a tempering process at $140{ }^{\circ} \mathrm{C}$ for the copolymer and at $110{ }^{\circ} \mathrm{C}$ for the terpolymer. This was done overnight in vacuo in order to remove residual solvent thoroughly and also made it possible to enhance the degree of crystallinity and thus the ferroelectricity. The force sensor was finalized by sputtering the customsized Au top electrode, in a similar fashion to the bottom electrode.

\section{3 Characterization and Instrumentation}

Firstly, ferroelectric properties were characterized. The two electrodes of the printed copolymer and terpolymer sensors were connected to the $20 \mathrm{kV}$ AC high-voltage amplifier (Trek Inc., New York) as well as the current amplifier (Stanford Research Systems. CA). In the case of printed copolymer sensor, a 100$\mathrm{mHz}$ sinusoidal wave was applied in the out-of-plane direction of the parallel-plate sensors with a maximum potential of $70 \mathrm{~V} / \mu \mathrm{m}$ from $20 \mathrm{~V} / \mu \mathrm{m}$ with increments of $10 \mathrm{~V} / \mu \mathrm{m}$. The maximum value was chosen since the printed copolymer sensors demonstrated electrical failure under an electric field slightly higher than 70 $\mathrm{V} / \mu \mathrm{m}$. During the measurements of each current-voltage cycle, the induced short-circuit current channel (peak-to-peak) was eventually maintained stable. The polarization was obtained after extraction of the leakage current which was related to the ion conduction in the materials. 
The capacitance $\left(C_{P}\right)$ and the dielectric behavior of the copolymer sensors were explored by broadband dielectric spectroscopy before and after the poling process, dealing with the same specimens as in the polarization process. The frequency range during the $C_{P}$ and $\varepsilon_{r}$ measurements varied from $0.1 \mathrm{~Hz}$ to $1 \mathrm{MHz}$. The sensors to be characterized were selected according to a normal dielectric profile; otherwise, both electrodes of the capacitor-like sensors were most probably interconnected during the Au electrode deposition.

The sensor performance was then investigated in response to the dynamic impact force. The wellprepared sensors with the top electrode stamped by a Teflon belt were mounted manually on the $10 \mathrm{~mm}$ thick PMMA panel via a cyanoacrylate adhesive. The dynamic force impulse was applied to the top of the printed copolymer and terpolymer sensors using an impact hammer (Dytran Instruments, Inc. CA), with a sensitivity of $1 \mathrm{~V} / 44.48 \mathrm{~N}(100 \mathrm{mV} / \mathrm{lbf})$ and a measuring gauge with the range $0 \sim 4448 \mathrm{~N}$. The applied force signal and current feedback response upon the both printed polymer sensors were recorded with the help of an Agilent oscilloscope. Afterwards, the charges were calculated by integrating current curves as a function of time and plotting them against the impact force pulse.

The sensitivity of the printed copolymer sensors was also investigated in response to the sweep signal with frequencies ranging between 100 and $1000 \mathrm{~Hz}$ (covering the frequency range of a typical impulse force) of the applied impact force. In a typical experimental setup, both printed polymer sensors were mounted at the free end of a vibrator (LDS, Brüel \& Kjær, UK), and were fastened with the standard mass weight of $10.0 \mathrm{~g}$ on the top Au electrode of the printed sensors. The sensor was short-circuit connected and consequently, the induced current in response to the force was recorded by an oscilloscope. In the meantime, a commercial MEMS-piezoelectric accelerometer (Brüel \& Kjær) was concurrently fixed to the experimental setup panel in order to monitor the in situ acceleration of the experimental assemblies.

In the case of the electrostrictive terpolymer, owing to a very slim hysteresis polarization loop and scarce remnant polarization, the impact force application could not yield a significant variation of the electric displacement. The impact force-induced charges would be reasonably ignored. Instead, the socalled pseudo-piezoelectric coefficient $d_{33 e q}$ could be determined under an applied electric field. A maximum of $240 \mathrm{~V}$ DC biased voltages was applied to the force sensor in the across section in two stepwise increments of $5 \mathrm{~V}$ below $30 \mathrm{~V}$ and $30-\mathrm{V}$ intervals above $30 \mathrm{~V}$ up to the maximum voltage value of $240 \mathrm{~V}$. The $d_{33 e q}$ coefficient under each electric field was then extracted.

The electromechanical behavior of the printed terpolymer sensors was also characterized in terms of electric field-induced strain and displacement in the thickness direction. Capacitor-like printed terpolymer sensor devices, with a sensing layer thickness of approximately $10 \mu \mathrm{m}$, were metallized with the help of a shadow mask and a 100-mHz sinusoidal AC voltage was applied upon the printed terpolymer sensor. The displacement was recorded by using the OFV-5000 Vibrometer equipped with an OFV-50x Sensor head (Polytec GmbH, Germany). A reflective paper was stuck in the center of the top electrode in order to measure the displacement in-situ. 


\section{Control of Printing Parameters for Optimization Process}

This section aims at providing a deep analyses on the control of several printing parameters that highly affect to the sample performance. Firstly, we address the printable droplet generated by the inkjet protocol which was tuned to adapt the ink formulation physics and rheology. Secondly, we discuss about the UVOzone treatment strategy allowing to efficiently eliminate the drop wettability issue of the fluid phobic PI substrate. Thirdly, we demonstrate that an optimal heating temperature and drop spacing are mandatory for the inkjet printing to be performed with a macrometer-scale patterns. A schematic illustration of the fabrication of a typical inkjet printed sensor will be also provided in order to better clarify this issue.

\section{1 Development of Jettable Ink Formulation}

Electroactive material deposition was realized by the DOD inkjet printing technology. The individual ink droplet was generated by a piezoelectric MEMS-modulated inkjet nozzle with an orifice dimension of $50 \mu \mathrm{m}$ diameter. The desired and patterned images of the polymer materials were inkjet printed by a precisely controlled ink formula deposition on the required location. It has been well recognized that a successful deposition of ink solution lies in a steady ink stream and a normalized droplet size, otherwise, vacant and imperfect polymer patterns are yielded which in turn affect the uniformity of the layers of residual polymers.

The printable ink formulation and microfluidic dynamics strongly determine the morphology and reproducibility of the droplets, in terms of viscosity $\eta$, as well as several pertinent physical factors such as the Reynolds $(R e)$ and Weber $(W e)$ numbers, etc. The feasible solvent is one of the essential requirements which should be primarily considered during inkjet printing of the polymer inks. Solvents for dispersing the colloidal suspensions or polymers are generally good solvent having the suitable surface tension, viscosity accordingly and right vapor pressure, otherwise, polymer crusts and finally clogging of the nozzle may occur [32]. The candidate solvents already used successfully for dissolving the PVDF-based polymer include methyl ethyl ketone (MEK) [19, 33], dimethylformamide (DMF) [34, 35], and triethyl phosphate (TEP) [36], and that these three solvents demonstrate a sharp contrast of boiling points. To a large extent, solvent evaporation most likely occurs for organic solvents having a relatively low boiling point. This might also lead to rather severe problems, such as a shrinking volume of the falling droplets, the interruption of the continuous ink ejection, nozzle clogging, etc. However, the use of a solvent with a very high boiling point would lead to a very slow evaporation of the polymer solution deposited onto the substrate. There is thus a remarkable trade-off between customized ink ejection and a highly efficient evaporation.

Table 1 Physical properties of the Dimatix cartridge, solvents and polymer ink solutions in TEP

\begin{tabular}{llllll}
\hline Physical parameters & $\begin{array}{l}\text { Dimatix } \\
\text { Cartridge }\end{array}$ & MEK & TEP & $\begin{array}{l}\text { Copolymer } \\
\text { inks }(1 \%)\end{array}$ & $\begin{array}{l}\text { Terpolymer } \\
\text { inks }(1 \%)\end{array}$ \\
\hline$\eta(\mathrm{cP})$ & $8-20$ & 0.43 & 1.46 & $2.16^{\mathrm{a}}$ & $3.26^{\mathrm{b}}$ \\
$\sigma\left(\mathrm{mNm}^{-1}\right)$ & $28-33$ & 24.6 & $30.6 \pm 3.0$ & 32.7 & 33.4 \\
\hline
\end{tabular}




\begin{tabular}{llllll}
\hline$\rho\left(\mathrm{g} \mathrm{cm}^{-3}\right)$ & $>1$ & 0.805 & 1.072 & 1.081 & 1.082 \\
b. p. $\left({ }^{\circ} \mathrm{C}\right)$ & -- & 79.6 & 215 & - & - \\
Pressure vapor $(\mathrm{Pa})$ & low & 10500 & 100 & - & - \\
\hline
\end{tabular}

${ }^{a, b}$ Note: values measured with a Brookfield viscometer according to the testing standard ASTM D 1824-1995 [37].

The continuous single droplet is also a result of the maneuverable viscosity of the polymer ink solution, which would be heavily adapted to the kinematic viscosity range of the nozzle specifications during the operation of ink ejection. Table 1 summarizes the physical specifications of the cartridge nozzle and two extensively used solvents including methyl ethyl ketone (MEK) and Triethyl phosphate (TEP) with a strong contrast of vaporization parameters. The MEK is too volatile to be employed as a jettable solvent, whereas the TEP has an adaptable vapor pressure. Furthermore, the macromolecular chain of the polymers can be stretched readily and precipitation can be avoided before reaching the very high critical polymeric concentration in an extensional flow [38].

Consequently, the copolymer and terpolymer inks were prepared using the TEP solvent, and the viscosities of both polymer inks were measured as $2.16 \mathrm{cP}$ and $3.26 \mathrm{cP}$, respectively, compared with 1.46 $\mathrm{cP}$ of pure TEP. These values were nicely adapted to the nozzle characteristics as well as the surface tension values. In order to generate a printable droplet, it is essential to understand the jettability mechanism. A successful ink droplet closely lies on the jet pulse waves and physico-chemical properties of the ink formula. The common dimensionless numbers, including the Reynolds $(\mathrm{Re})$, Weber $(\mathrm{We})$, Capillary $(\mathrm{Ca})$ and $Z$ numbers, which are relevant to the droplet formation dynamics, are governed by the inertial force, the viscosity, the surface free energy, etc., presented as follows [39-42]:

$$
\begin{aligned}
& R e=\frac{\text { inertial } \text { force }}{\text { viscous force }}=\frac{\rho v d}{\eta} \\
& W e=\frac{\text { inertial force }}{\text { suface tension }}=\frac{\rho v^{2} d}{\sigma} \\
& C a=\frac{\text { viscous } \text { force }}{\text { surface tension }}=\frac{\eta v}{\sigma} \\
& Z=\frac{\sqrt{\sigma \rho d}}{\eta}=\frac{1}{O b}
\end{aligned}
$$

where $\sigma, \rho$ and $\eta$ symbolize respectively the surface tension, density and viscosity of the ink formula. Here, $v$ is the initial velocity of the high shear rate ink flow pumped out of the nozzle with diameter $d$.

However, when the piezoelectric-driven actuator is triggered by the voltage pulse waveform, the polymer solution ink is expelled out of the nozzle with a large initial velocity. Its behavior during the inkjet printing process matches the characteristics of viscoelastic fluidic jets, according to the ejection behaviors of a non-Newtonian fluid ink [43, 44]. A series of photographic snapshots of an ink solution were taken during the ink ejection as a function of time with the help of a stroboscopic video camera (see Fig. S1 in supporting information). The droplet formation was maintained through a short and thick ink filament 
attaching to the nozzle at the initial stage of ink ejection. The filament became longer and thinner as the time elapsed. After several microseconds, the droplet detached from the nozzle at the occurrence of an abrupt collapse of a liquid ink ligament. The elongated tails resistant to break remained attached to the primary bulk droplet through the thinning ink filament at around $260 \mu$ s and finally a single and spherical droplet bead was generated. This drop formation of an elongational flow was essentially quite far from a Newtonian fluid, of which the liquid filament was meant to be easy to break and destabilize into a sequence of satellite small droplets behind the main polymer drop[45-47].

A clear train of the satellites behind the bulk droplets was observed for both the copolymer and terpolymer ink solutions, which successively retracted into the primary bulk droplet during a time of flight of $260 \mu \mathrm{s}$ (also see Figure S1 snapshot graphics of supporting information). The coalescence of these satellites with the primary droplet was acknowledged due to the surface tension, viscosity and density of the ink solutions [48].

It is well-known that all printable pure solvent systems were considered to be Newtonian because of their simple molecular structures and indeed showed nearly Newtonian behavior over the experimental ranges of importance. To the best of our knowledge, the addition of nanoparticles or polymers generally increases an ink's low shear rate viscosity. At sufficiently high particle loading ( $\sim 60 \%$ mass loading) can lead to non-Newtonian behavior, such as shear thinning at moderate shear rates and shear thickening at very high shear rates if significant hydrodynamic coupling arises between particles. A small amount of highmolecular-weight polymer dissolving in solvent attends to dramatically alter the rheological behavior from a nearly Newtonian fluid to a non-Newtonian one showing viscoelastic effect and an increase in elongational elasticity. However, the Newtonian behavior, i.e., the rare satellites that accompanied the primary droplets, could be no longer seen if the polymer concentration reached this critical concentration threshold. In fact, the concentration was a key parameter in the physical behavior of the polymer ink solution. The disintegration of ligaments was unlikely to occur at relatively high polymer concentration, which corresponds to a very typical non-Newtonian fluid behavior, with a long liquid tail of ink flow following behind the primary bulk droplet bead[49]. In this circumstance, a sequence of ink beads was created because the surface tension of the polymer ink solutions was too weak to retract the free elongated ink flow. In this study, the engineered copolymer and terpolymer inks exhibited a typical non-Newtonian behavior at a polymer concentration of $1 \mathrm{wt} \%$, thereby the dynamic viscosity at high shear rate was most likely to be depressed. Unfortunately the viscosity of the polymer solution at very high shear rate wat yet to be available for the moment. However, the viscosity measured at low shear rates can still be employed as a reference to calculate the dimensionless parameters, $R e, W e$, etc of the ink liquids at high shear rates in the tubes because the viscosity of the both polymer solutions did not strongly differ from that of the pure solvent at high shear rates.

As a matter of fact, the physics and pressure of the inks, the nozzle orifice diameters and the electric waveform pulse affected the physics of the droplets. The droplet velocity and volume were monitored via a stroboscopic vision system mounted on the inkjet printer. The physical constants of the inkjet printing criterion were calculated according to equations 8-11 (see Table S1 in Supporting Information). Since the 
parameters, except the viscosity, did not vary from the pure solvent-based ink to the polymer ink solutions, the rheological behaviors of the inks could be regarded as a key role for determining the jettability. The derived $\mathrm{Z}$ value was indicative of the jettability where a low $\mathrm{Z}$ value reflects that the satellites may be created in parallel with the main ink droplets $[8,50]$. In the case of copolymer inks with relatively low viscosities, a high $\mathrm{Z}$ value of 61.54 was found, which was out of the reported printability range of $1<\mathrm{Z}<60$ [49] previously determined, although the rheological properties and printing protocol presently satisfied the requirement of a stable inkjet printing. On the other hand, the higher viscosity of the terpolymer inks led to a $\mathrm{Z}$ value of 42.22 which matched nicely the defined printable limits and thus gave rise to a very stable jetting droplet, as could be expected. It should also be noted that pure TEP-based inks presented a matched printability under the optimal printing protocol even though the $\mathrm{Z}$ values were as high as 91.04, which was indeed out of the defined printability range $[41,49,51,52]$. These results suggested that pure TEP and TEP-based polymer ink solutions were robust and extended the range of printability for the inkjet printing technology.

\subsection{Surface Modification}

Once the ejected polymer is deposited onto the substrate, new challenges arise. When the drops of polymer solution inks on the substrate start to dry, the concentration of either the polymer solute or the suspended particulate matter gradually increases during the solvent losing whereafter predetermined images are patterned until the deposited polymer is solvent-free. If the solvent drying process is not properly controlled, well-known issues such as the coffee ring effect and consequently inkjet printed films with inhomogeneous thicknesses would occur. Although some researchers have fabricated functional devices with the help of this effect [53-56], an uneven topological behavior of inkjet printed films has an extremely deleterious influence on the performance and lifetime of inkjet printed electronics and electroluminescent devices [57].

This issue is also of importance in present inkjet printing process when the ink droplets dry, and must be avoided or solved. Additionally, a quick and large portion of ink deposition results in a significantly uneven thickness distribution, giving rise to topological wrinkles and eventually inkjet printed electronics with tilted defect. It would hence be interesting to investigate the compatibility between the ejected materials and the polymer substrates, where the wettability has a vital role.



Figure 2 Schematic illustration of a layer-by-layer configuration of both inkjet printed electroactive polymer sensor 
The surface tension of a solid substrate, serving as a quantitative reference of the wettability, would benefit the desired patterns during the drying process. In this context, the fabrication flow should be considered. Figure 2 represents a schematic diagram of inkjet printed copolymer and terpolymer sensors, where the sensing polymer layer binds to both the PI substrate and the sputtered Au electrodes. The measured surface tension values $\sigma$ of the pristine PI foil were almost equivalent to the value of the Au electrode, and quite close to that of the solvent-free as-printed polymeric thin layer. During the drying of the inks, the $\sigma$ values of drop layers increased from the surface tension threshold of the dilute polymer ink solutions. The wettability was improved with the help of UV-Ozone exposure to the pristine PI foil. The treatment effects could be reflected directly by the water droplets on the PI foils (see Figure S3, supporting information). The contact angle, shown in Figure 3, dropped from $71.17^{\circ}$ on the pristine surface down to around $25^{\circ}$ with only $5 \mathrm{~min}$ of UV-Ozone exposure, and this corresponded to a surface tension value of $71.38 \mathrm{mN} \mathrm{m}^{-1}$, which was much larger than the surface tension values of the polymer inks and for both inkjet printed copolymer and terpolymer layers which were respectively $39.4 \mathrm{mN} \mathrm{m}^{-1}$ and $58.01 \mathrm{mN} \mathrm{m}^{-1}$. A rather low contact angle is indicative of good adhesion between the PI foil and the solidifying polymer during the volatilization of the TEP solvent, although an excellent interface adhesion was never demonstrated in the present work.

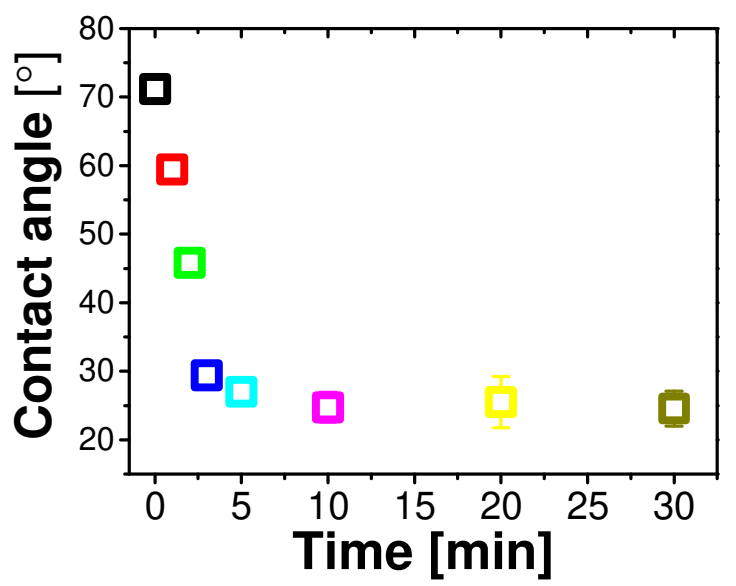

Figure 3 Water contact angles on the PI foil substrate as a function of the UV-Ozone exposure time. The error bars are overlapped with the square symbols.

\subsection{Inkjet-Printed Film Profiles}

The aforementioned coffee ring effect was a ubiquitous phenomenon even though the wettability was excellent [58]. Particularly in the case of the present inkjet printing, there existed a strong contrast regarding the wettability between the UV-Ozone treated PI foil and the Au electrode. The evaporation rate at the edges of already fallen droplets exceeded the solvent volatility in the droplet center and this led to a large amount of the solvent being lost at the droplet edges close to the three-phase contact line. This contact line was however pinned so that the lost liquid could be replenished by an outward capillary flow from the 
interior of the droplet. This convective flow carried with it dispersed polymer particles that piled up at the contact line, thus leading to the coffee ring effect.

This outward flow can however be balanced by an inward Marangoni flow towards the droplet interior, driven by a surface tension gradient between the periphery part and the interior of the drying droplets. Several strategies have been developed to overcome the coffee ring effect in terms of ink formulation, surfactant additives [59,60] and heating conditions [61]. The main approch invloved engineering the inward Marangoni flow by a cosolvent strategy, with one minor component posessing a high boiling point and a relatively low surface tension [62-64]. This strategy has worked very well for printing patterns detailed in the litterature $[65,66]$. However, for the potential electric sensor device application in the current study, the introduction of additives into the formulation was ruled out.

Alternatively, an inward Marangoni flow can be enhanced through printing protocols instead of the cosolvent approach because the latter approach tends to change the rehological behavior of the inks. This technique was thus carried out, with large-area printed functional films rather than isolated single ink droplets which consistently suffered from inhomogeneous film morphologies due to the printng sequence. In other words, droplet coalescencee along the $\mathrm{x}$ axis led to the formation of a line and when the line coalesced with those next to it, a film was formed. In this circumstance, additional printing parameters must be taken into account. By controlling the spacing between droplets and the heating temperature, the coffeering effect in bulk film printing patterns could be minimized with a single-solvent inkjet printing solution in the present study.

Subramanian et al. [67] reported on a perfect morphology of inkjet-printed PEDOT:PSS lines under controlled printing protocols and temperature couditions in terms of drop spacing, firing frequency and substrate heating temperature and they also provided an explanation from the viewpoint of printing geometrics. The drop spacing, i.e., the distance between two adjacent droplets' centers, is linked to the pattern resolution. Decreasing the drop spacing is equivalent to increasing the pattern resolution. For the commercially compact inkjet printer employed in this work, the low resultion was automatically compensated by ejecting excessive amounts of ink solution in order to fully cover the drop spacing in a pragmatic printing assignment. Large portions of ink deposition prolonged the time required for solvent evaportion, as the main side-effect of a low printing resolution. 


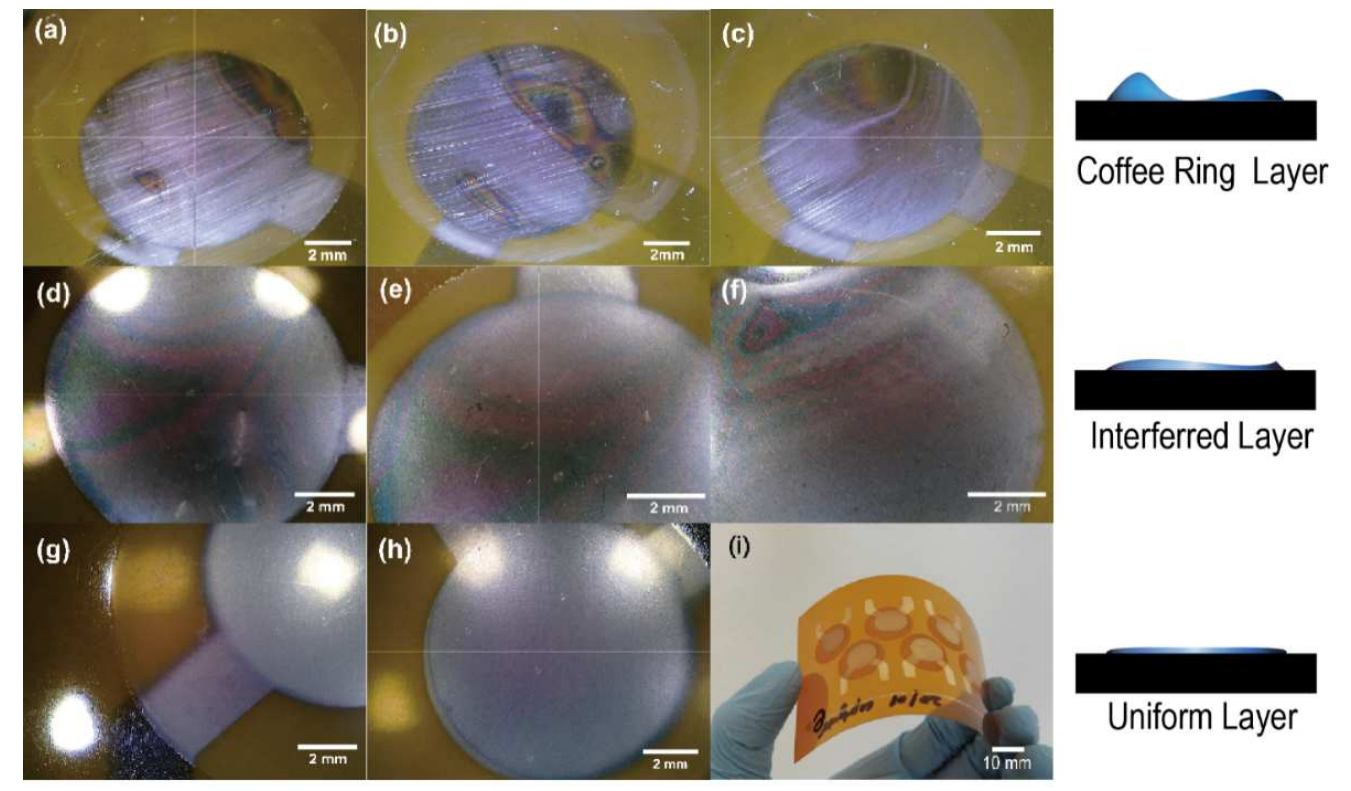

Figure 4 Optical photographs of inkjet-printed terpolymer films on a UV-Ozone-treated Kapton substrate with varying heating temperatures. (a)-(c) Discrete and incomplete terpolymer layer at $70{ }^{\circ} \mathrm{C}$; (d)-(f) colorful interplay strips due to thickness discapancies of the polymer layer at $80^{\circ} \mathrm{C} ;(\mathrm{g})-(\mathrm{h})$ strip-free and optically homogeneous topologies at a heating temperature of $90^{\circ} \mathrm{C}$; (i) monolithically inkjet-pritined force sensors. The horizontal bar at the bottom right of each image shows the scale.

This negative effect becomes exagerated when the printing process was repeated a second time, resulting in a severe coffee ring pattern. The best strategy was the following: with a certain drop spacing the ink droplet merged with the adjacent pinned beads and in parallel, the deposited ink dried almost when the upcoming repetition of the printing was about to launch. As motivated in this case, the substrate temperature played a vital role during the drying process of the pinned droplets. In the printing trials, the substrate temperture was set between $70^{\circ} \mathrm{C}$ and $90^{\circ} \mathrm{C}$, and the temperature dependence of the morphologies of bulk patterns is presented in Figure 4. The heating at $70^{\circ} \mathrm{C}$ took much longer than that at $90{ }^{\circ} \mathrm{C}$. Decreasing the substrate temperature signifcantly reduced the evaporation rate of the inks at the periphery of the drops more than in the center. In this regard, the liquid drop was maintained on the substrate before the next droplet fell. Implementation of ink ejection as a secondary repetition could give rise to bulging because of the ink overflowing. On the other hand, a high substrate temperature favored a high evaporation rate of the pinned droplet, for which the drop spacing needs to be optimized. The evaporation rate per area $J_{(r)}$ correlates to the $(R-r)^{-\lambda}$, where $\lambda$ is obtained from the contact angle $\left(\theta_{c}\right)$ between the drop and the substrate as $\lambda=\frac{\pi-2 \theta_{c}}{2 \pi-2 \theta_{c}} ; r$ represents the distance from the drop center to its periphery; $R$ and $\theta_{c}$ are the pinned drop radius and the contact angle, respectively. The evaporation flux both at the center and rim of the pinned drop were enhanced so that the in-situ viscosity of the pinned drop multiplied before the upcoming adjacent droplets landed. It would be reasonable to assume that the onset of scalloping can be observed at relatively low drop spacing. Hence, the coffee ring effect in the bulk film was effectively minimized because of a weakening of the outward capillary flow in an isolated single ink droplet. 


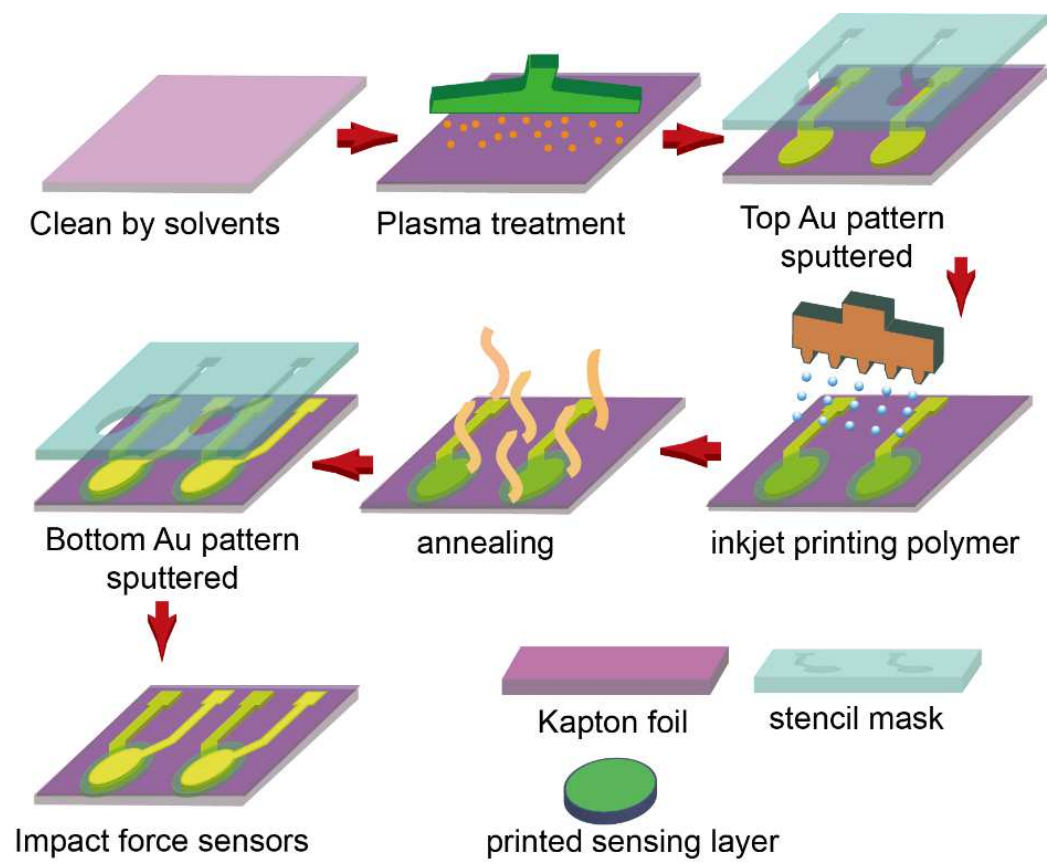

Figure 5 Schematic illustration of the fabrication of a typical inkjet-printed electroactive polymer sensor device

\section{Sensor performance - Results and Discussion}

This section focusses on the sensor behavior as well as the dynamic impact force based on electrical characterization of two different printed materials comprising the copolymer $\mathrm{P}(\mathrm{VDF}-\mathrm{TrFE})$ and the terpolymer P(VDF-TrFE-CTFE). The sensing response of both materials will be compared and evaluated via several relevant factors like dielectric strength, capacitance, Young modulus, and (pseudo)piezoelectric constant. For a better understanding of each material performance, a frequency analysis of an intrinsic material parameter like the permittivity is also investigated.

\section{1 Characterizations of electrostrictive and ferroelectric materials based on Inkjet Printing}

The ferroelectric properties of inkjet-printed copolymer sensors were characterized by their displacement vs the hysteresis in a bipolar electric field. High electric fields up to $70 \mathrm{~V} / \mu \mathrm{m}$ with increments of $10 \mathrm{~V} / \mu \mathrm{m}$ from $20 \mathrm{~V} / \mu \mathrm{m}$ were applied to the inkjet printed copolymer sensors in order to enhance the ferroelectric performance through aligning the flipping of the dipoles with the electric direction. The output of the short-circuit current was maintained stable during each poling cycle. The displacement of the ferroelectric P(VDF-TrFE) copolymer sensors under an alternating electric field, as presented in Figure 6, showed a polarization curve typical to that of a ferroelectric ceramic $[68,69]$. The polarization reached its saturation under a maximal poling electric field of around $70 \mathrm{~V} / \mu \mathrm{m}$, above which a high risk of mechanical failure occasionally took place due to the high electric field. 
The remnant polarization $P_{r}$ when the electric field was switched off and for the coercive field averaged at around $65 \mathrm{mC} \mathrm{m}^{-2}$ and $45 \mathrm{~V} / \mu \mathrm{m}$, respectively, and these values were close to those reported for a pressure-sensor based on the $\mathrm{P}(\mathrm{VDF}-\mathrm{TrFE})$ copolymer [70, 71]. An excellent reproducibility was found by the electric hysteresis measurement for each fabrication batch, suggesting a homogeneity in the thickness of injet printed terpolymer layers as well as the layers' uniformity thanks to the DOD inkjet printing technique. The hysteresis behaviors of the electrostrictive P(VDF-TrFE-CTFE) terpolymer were also characterized but an incomplete hysteresis loop was unfortunately obtained, indicating considerable losses of the devices during the capacitor discharge.



Figure 6 Ferroelectric hysteresis displacement (D) vs. electric field (E) of the inkjet printed copolymer sensor.

Measurements of the capacitance $(C p)$ at low bias voltage $(\sim 0.1 \mathrm{~V} / \mu m)$ as a function of the broadband frequency $f$ were also carried out by an impedance analyzer for both the poled ferroelectric copolymer sensors and the electrostrictive terpolymer sensors. The representative spectra of the logarithmic $f$ dependence of $C p$ are plotted in Figure 7a. The mean $C p$ value of the ferroelectric copolymer showed a slight drop as a function of the frequency range, with a plateau averaging at $0.72 \pm 0.03 \mathrm{nF}$ at medium $f$ range. The values found for the inkjet printed copolymer sensors were close to the $C p$ values for the customsized sensors which were fabricated by spin-coating and screen-printing. Regarding the electrostrictive terpolymer sensor, the capacitance surprisingly decreased from $12.12 \mathrm{nF}$ at low $f$ to a value leveling off at $3.30 \pm 0.21 \mathrm{nF}$ at medium $f$, approximately 5 times higher than the $C p$ value of the copolymer sensors.

The dielectric behavior derived from expression $\varepsilon^{\prime}=\frac{C_{P} \cdot d}{\varepsilon_{0} \cdot A}$ shared common features with the capacitance properties. Here, $d$ corresponds to the thickness of the electroactive polymer layer; $A$ is the electrode area; and $\varepsilon_{0}$ is the permittivity in vacuo. The inkjet printed copolymer sensor presented a typical dielectric spectra versus $f$ as discussed in the established reports dealing with the sensor devices fabricated via the versatile printing technique, whereas for sensors based on the electrostrictive terpolymer, there was an exceptional enhancement at low frequency as compared with the terpolymer dielectrics based on the spin-coating, see Figure 8a. In order to clarify this eccentric phenomenon, dielectric loss spectra were 
obtained after subtraction of ion conduction contribution using the well-known Kramer-Kronig transforming method.

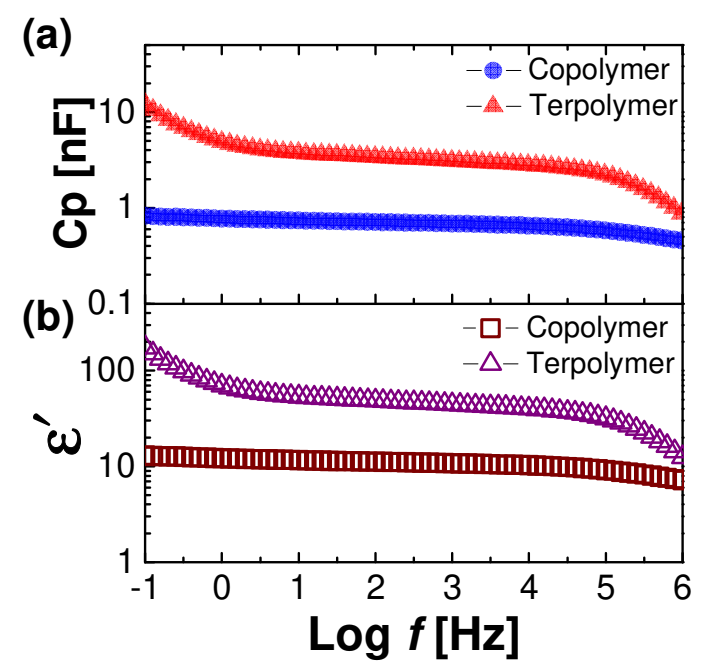

Figure 7 Logarithmic frequency dependence of the capacitance (a) and dielectric permittivity (b) for inkjet-printed copolymer and terpolymer sensors. The filled and open square denote the frequency dependence of the capacitance and the dielectric spectra for the P(VDF-TrFE) copolymer sensors. The filled and open triangles represent the data obtained for the terpolymer sensors.

The dielectric loss curves before and after transformation are plotted in Figure 8b. The dielectric loss derivatives $\varepsilon_{d e r}^{\prime \prime}$ exhibited significant discrepancies over the spin-coated film at low frequency, as opposed to for the medium to high frequency. We have revealed the high dielectric amplitude at low frequency was satisfactorily ascribed to the interfacial polarization $[18,66]$. The surprisingly high dielectric loss for the inkjet printed terpolymer sensor implied an evolution of such relaxation over the spin-coated one. The results further showed that a peak shift of more than one decade towards higher frequencies occurred for the dielectric modulus of the inkjet printed terpolymer sensors. This might be due to a unique polymer ink deposition via the inkjet printing technique, differing from that of spin-coating layers where the deposition of ink solution was physically continuous. Scalloped or uniform patterns were realized rather than bulging or coffee ring patterns due to the overflowing ink deposition. In this case, each pinned droplet dried individually on the substrate, which led to relatively isolated crystal nano-domains instead of a continuous polymer microstructure via a non-optimized inkjet printing protocol or spin-coating method. Each relatively isolated microstructure serving as a micro-capacitor would strengthen the boundary effect, namely, the MWS interfacial polarization. 


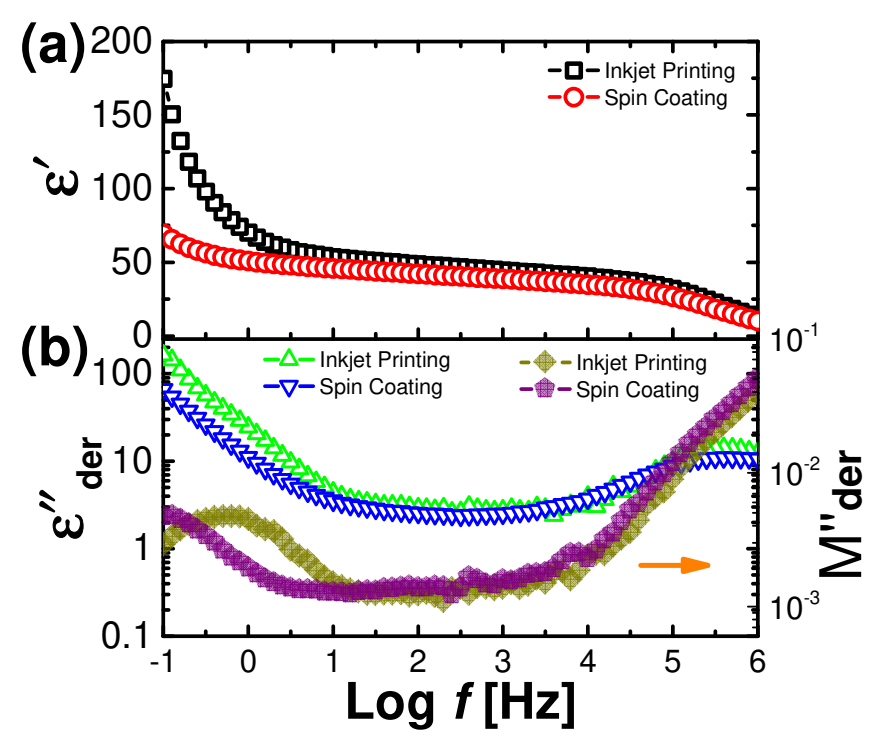

Figure 8 A comparison of inkjet printing and spin-coating protocols with respect to broadband dielectric spectroscopy (a) and the dielectric loss derivative $\varepsilon_{d e r}^{\prime \prime}$ with dielectric modulus $M_{d e r}^{\prime \prime}$ spectra as a function of the logarithmic frequency for terpolymer material

\section{2 Piezoelectric Behavior of Inkjet-Printed Copolymer Sensor}

The piezoelectric performance of a single ferroelectric force sensor was investigated with a varying dynamic force application. Prior to the evaluation of the force sensor performance, the sensitivity of the force sensor was characterized. The dynamic force with varying frequencies were applied on the top of the sensor surface by means of a frequency-modulated vibrator. The reference acceleration of the $\mathrm{z}$ axis direction was monitored in-situ by a commercial MEMS-accelerometer bound close to the inkjet printed copolymer sensor on the polycarbonate panel, which was mounted on the free z-axis end of a vibrator (as shown in Figure 9). The real-time force applied on the inkjet printed copolymer force sensor was defined by the standard weight mass $(10.0 \mathrm{~g})$ multiplied with the acceleration values that was able to be modulated via the changing parameters of the vibrator in terms of oscillating amplitude and frequency of an input source. The sinusoidal signal with a frequency of $100 \mathrm{~Hz}$ up to $1000 \mathrm{~Hz}$ in increments of $100 \mathrm{~Hz}$ was successively applied to the vibrator. The electric circuit for measuring the sensitivity is schematized in Figure 10. The representative output current of the inkjet printed copolymer force sensor in response to the applied force at a given frequency of $400 \mathrm{~Hz}$ is illustrated in Figure 11a. Note that the simultaneous output current featured a quasi-sinusoidal feedback signal. And interestingly, a phase shift between the applied force signal and the yielded current was clearly observed. Such a phase shift coincided with the theoretical model of equation 7 since the output current was proportional to the time derivative of the strain $\left(\partial \mathrm{S} \_33\right) / \partial \mathrm{t}$ in thickness mode [67]. The mechanical strain does not always align with mechanical excitation because of the viscoelastic behavior of the polymer [68]. 




Figure 9 A photograph of the experimental assembly measuring the sensitivity of the inkjet printed copolymer force sensors

An investigation of the piezoelectric behaviors of the printed copolymer sensor vs. the applied force was also carried out at a fixed frequency of $400 \mathrm{~Hz}$. Note that the output current in Figure $11 \mathrm{~b}$ was a quasilinear relation with an applied force of no more than $1 \mathrm{~N}$. Since a reliable setup for evaluating the sensing device at high accuracy was presented, the peak-peak amplitude of acceleration and the current response curves were both taken as data for the sensitivity calculation of the printed copolymer force sensor. In addition, the sensitivity of the printed copolymer force sensor as a function of the frequency at a fixed mechanical excitation amplitude of the input sinusoidal signal was then plotted in Figure 12. It can be clearly observed that the sensitivity, the specific ratio of the readout current to the varying applied force values at each studied frequency, averaged at $11.8 \pm 1.1 \mathrm{nA} \mathrm{g}^{-1}$ except for an anomaly. The anomalous region in the sensitivity demonstration appeared at a frequency between $150 \mathrm{~Hz}$ and $250 \mathrm{~Hz}$, which was unambiguously ascribed to the resonance frequency of the experimental setup. This corroborates the idea of the sensitivity of the printed copolymer sensor not depending on the physical features of the applied force in terms of frequency.

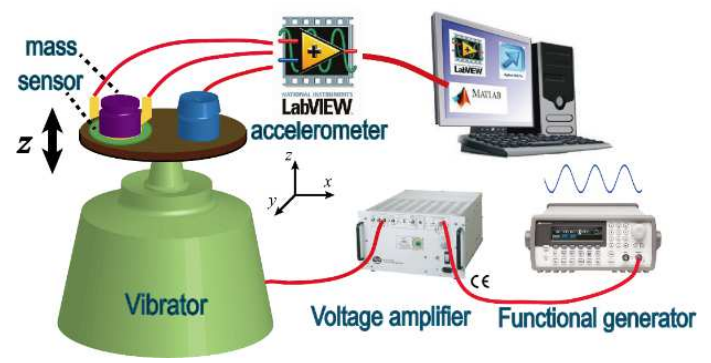

Figure 10 The illustration of the experimental assembly for the sensitivity measurement of the inkjet-printed copolymer force sensor. 

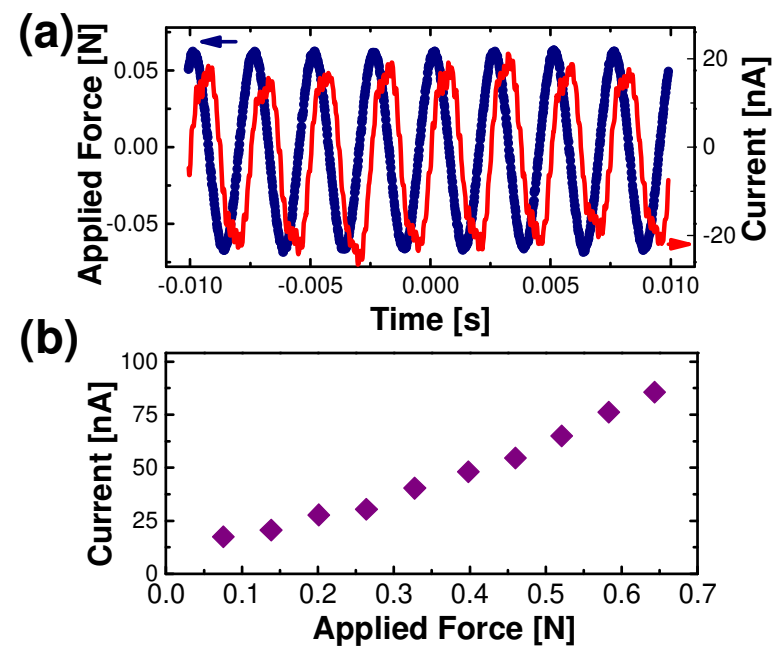

Figure 11 A representative feedback current in response to a given applied force of $400 \mathrm{~Hz}$ as a sinusoidal signal (a); output current $I$ against the dynamical force application for a single printed copolymer sensor at a fixed frequency of $400 \mathrm{~Hz}(\mathrm{~b})$.

Validation of the printed copolymer sensor was manifested by the dynamic force impulse application with a schematic illustration of the experimental setup in Figure 13a. The applied force impulse profiles featuring a Gaussian-shaped mechanical excitation signal are shown in Figure 13b. The force peak appeared at $5.76 \mathrm{~V}$ which corresponded to the maximal dynamic force of $255.78 \mathrm{~N}$ excitation. The feedback current and charge response of the printed copolymer sensors to such a force were then illustrated accordingly where the output signal showed the occurrence of a sinusoidal current output and consequently, a charge yield of $4.77 \mathrm{nC}$ was obtained via the integration of the feedback current profile versus time. It is also worth noticing that the shape of the charge and the corresponding peak was shared with that of the applied force impulse, indicating that the yield charge was consistent with the applied force. Hence, the piezoelectric coefficient $d_{33}$, defined as the ratio of the charge to the applied force, was calculated as $18.7 \mathrm{pC} \mathrm{N}^{-1}$, quite close to the best values $\left(\sim 20 \mathrm{pC} \mathrm{N}^{-1}\right)$ reported in the literature $[6,70,72]$.

A varying force impulse was then applied to the printed copolymer sensor in a range from $50 \mathrm{~N}$ to 305 $\mathrm{N}$. The charge in response to such a dynamic force was plotted in Figure $13 \mathrm{c}$ and was linearly fitted with a slope value of $18.9 \mathrm{pC} \mathrm{N}^{-1}$. Note that the frequency of the applied forces was estimated at approximately $300 \mathrm{~Hz}$ which was not within the measured resonance range $(150 \sim 250 \mathrm{~Hz})$. The replicate measurements were independently carried out with a batch of six sensors and the variation of $d_{33}$ among the individual sensors was negligible and statistical, which in turn implied a homogeneity and reliability of the printed copolymer sensor. 




Figure 12 Sensitivity of the printed copolymer sensor against the applied dynamic force with frequency profiles ranging from $100 \mathrm{~Hz}$ to $1000 \mathrm{~Hz}$. The solid curve is a peak fit according to the Lorentz force law. The hatched area denotes the mechanical resonance region of the experimental setup.

(a)
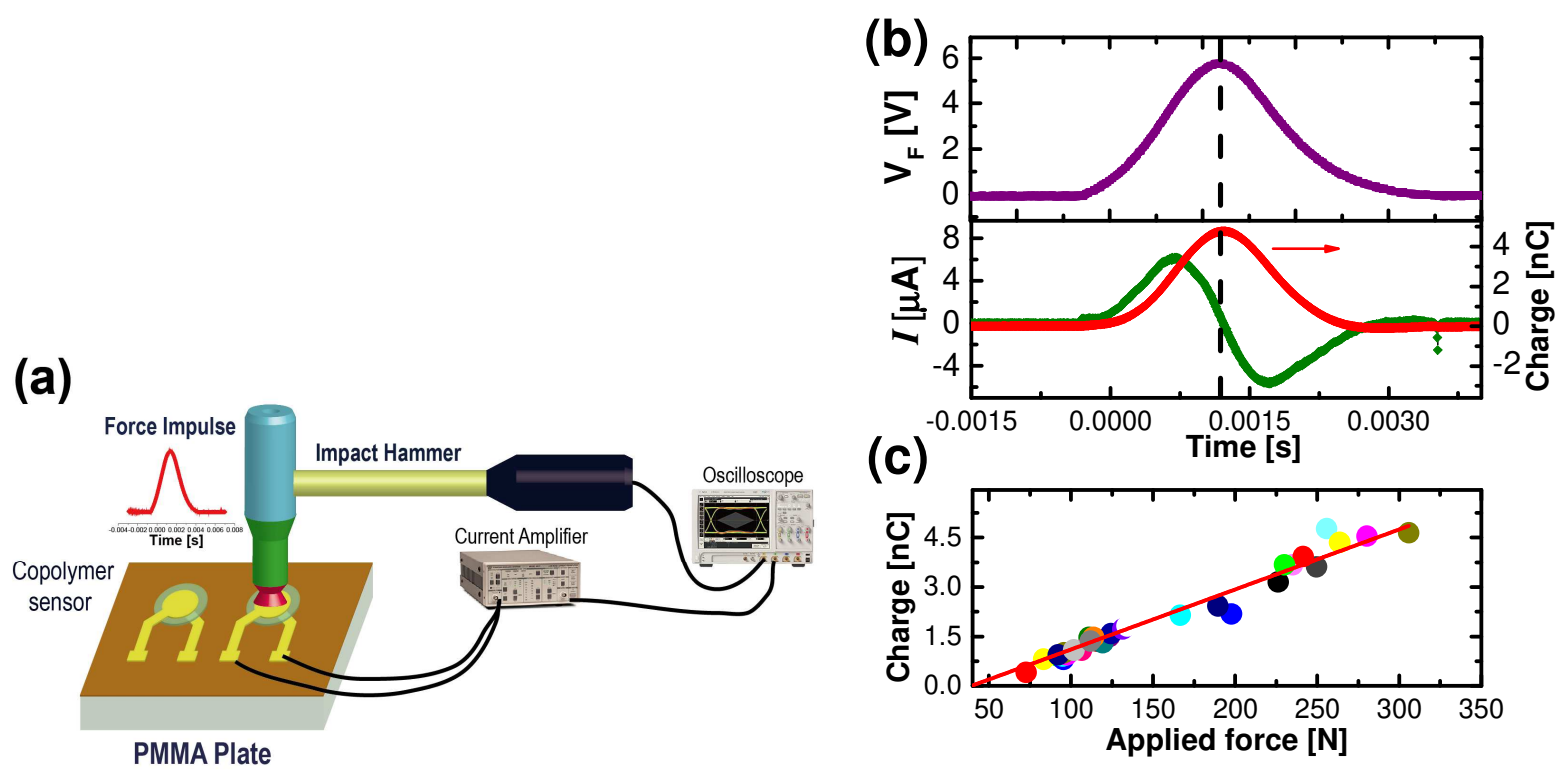

Figure 13 Schematic illustration of the experimental setup measuring the impact force upon the inkjet printed P(VDF$\mathrm{TrFE}$ ) copolymer force sensor device (a). Evolution with time of the voltage trace of the applied dynamic force impulse and the current feedback $I$ with integration of the charge (b); Induced charge as a function of the applied dynamic force. The solid line is a linear fit to the data (c).

\section{3 Pseudo-Piezoelectric Behavior of Inkjet-Printed Terpolymer Sensor}

As motivated by the electric polarization hysteresis behavior from a theoretical point of view, the large electric polarization of the electrostrictive terpolymer could be induced under the bias electric field $E$ as already stated in the section 2. Moreover, its so-called pseudo-piezoelectric coefficient $d_{33 e q}$ was linearly dependent on the bias electric field $E_{33}$ across the sensor thickness direction. In order to validate such a 
hypothesis, impact force excitations upon the printed sensors based on the terpolymer were characterized under varying electric fields up to $33.33 \mathrm{~V} / \mu \mathrm{m}$, corresponding to a bias voltage of $300 \mathrm{~V}$. Its measuring circuit is illustrated in Figure 14.

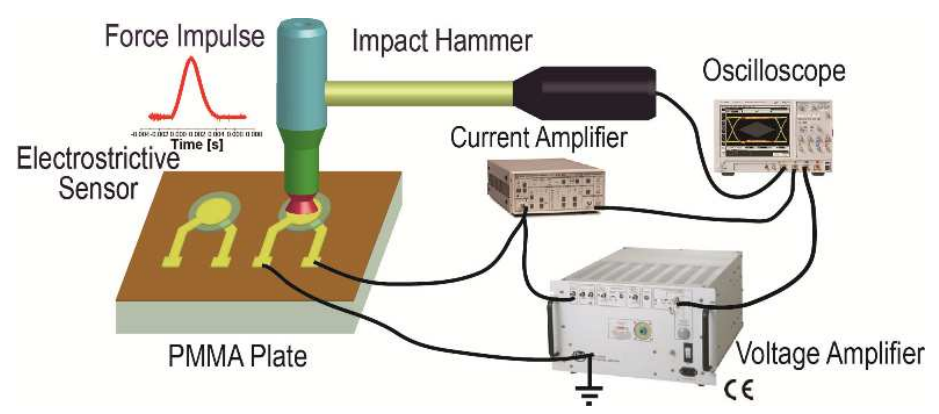

Figure 14 Schematic illustration of a dynamic force impulse upon the inkjet printed terpolymer sensors based on the electrostrictive terpolymer and characterization circuit.

The representation of the feedback current and charge profiles of the dynamic force stimuli under the bias voltage of $10 \mathrm{~V}$ and $90 \mathrm{~V}$ is plotted in Figure 15. Interestingly, the shape of the current and charge output curves demonstrated the coincidence with those of the printed copolymer sensor. It was comparable
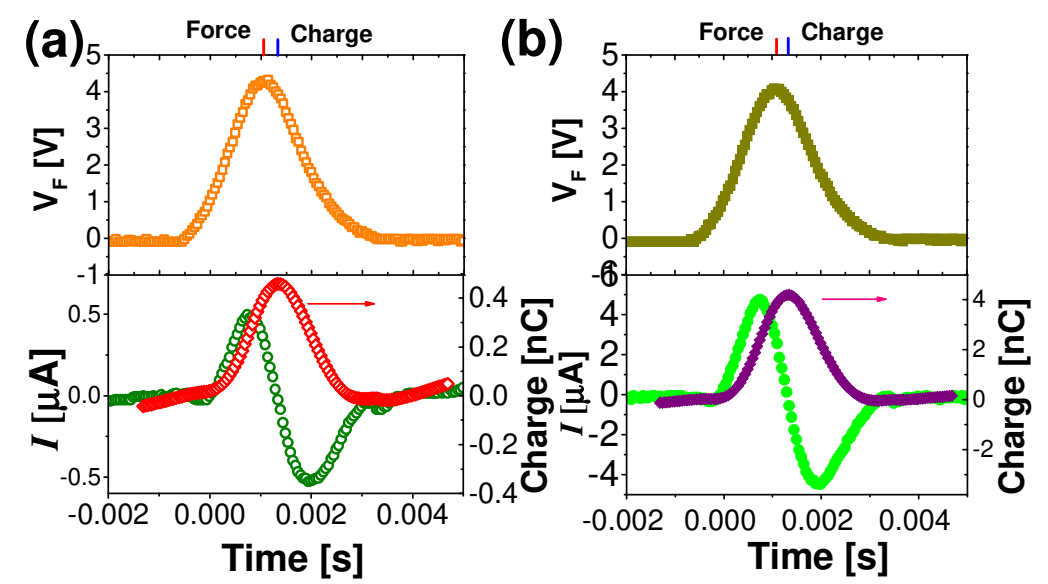

Figure 15 Representation of readout current and integration of charge in response to an equal dynamic force under bias electric fields of $10 \mathrm{~V} / 9 \mu \mathrm{m}$ (a) and $90 \mathrm{~V} / 9 \mu \mathrm{m}$ (b).

to that of the terpolymer sensor under $90 \mathrm{~V}$ bias voltage presenting a charge of $4.27 \mathrm{nC}$, nearly 9 times higher than the charge response of $0.46 \mathrm{nC}$ under $10 \mathrm{~V}$ bias voltage when a similar dynamic force excitation $(185.4 \mathrm{~N})$ was applied. It should also be noted that a 9 times higher bias voltage resulted in a nearly 9-fold charge yield and it thus was reasonable to assume that the pseudo-piezoelectric coefficient $d_{33 e q}$ relied significantly on the bias voltage. To furthermore corroborate this observation for the inkjet printed terpolymer sensors, the charges of current integrations were plotted at increasingly selected DC bias voltage as a functional of input dynamic force in Figure 16. The induced charges for a given DC bias voltage, followed a quasi-linear fit to the impact force, suggesting good reliability of the printed terpolymer force sensor. On the other hand, the slope of the charges vs. impact force fitting curves, routinely defined as the 
pseudo-piezoelectric coefficient was greatly enhanced as the increase in DC bias voltage, indeed consistent with the theoretical consideration in section 2 where the modeling pseudo-piezoelectric coefficient $2 M_{33} E_{3}$ was the linear function of DC bias electric field. Because of rather good reliability presented by the electrostrictive terpolymers, sensor and actuator devices based on this polymer and its composites have been demonstrated in our group and revealed the high stability of the sensor for electrical input range [30, $73,74]$.

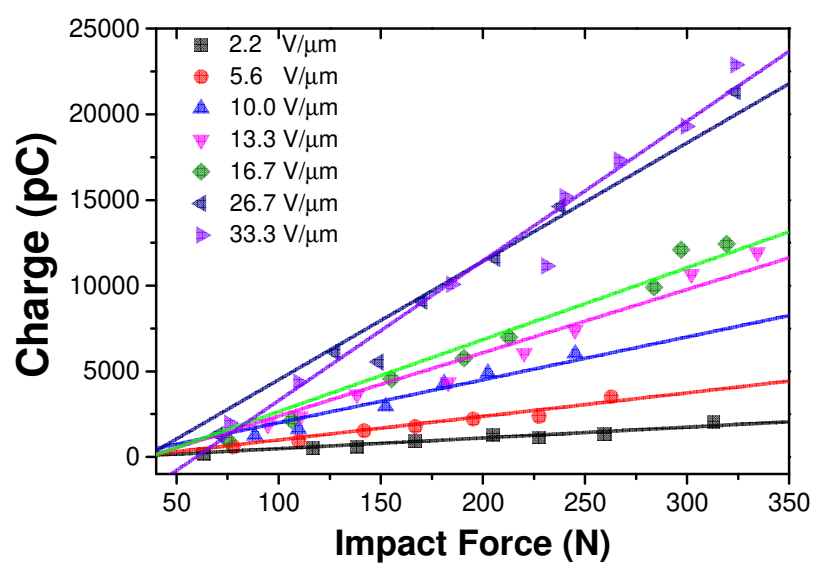

Figure 16 Plots of induced charges as a function of dynamic impact force upon the inkjet printed terpolymer sensor under varying DC bias voltage showing a quasi-linear fitting feature

Furthermore, individual $d_{33 e q}$ value at each bias voltage were subsequently extrapolated and increased monotonically with the bias electric field. This behavior could be satisfactorily described with a quasi-linear fitting law as shown in Figure 17. This behavior could be satisfactorily described with a quasi-linear fitting law as shown in Figure 17. A $d_{33 e q}$ value under $33.33 \mathrm{~V} / \mu \mathrm{m}$ was equivalent to a $300 \mathrm{~V}$ bias voltage, leading to a pseudo-piezoelectric value of $81.64 \mathrm{pC} \mathrm{N}^{-1}$, nearly 4 times higher than that of the printed copolymer force sensors. The piezoelectric response of the ferroelectric polymer and the ceramic has also been added on the Figure 17 for facilitating the comparison. The objective is not to put them in competition but to show the complementarity of each technologies. It has been seen in Figure 17 that the terpolymer exhibited substantial piezoelectric coefficient that was comparable to the one of the ceramics for an electric field of around $40 \mathrm{~V} / \mu \mathrm{m}$. In fact, over the last few decades, much attention has been paid to the study of organic ferroelectric materials. These electroactive polymers are very attractive because of their light weight, easy molding and good piezo/pyroelectric properties with respect to the inorganic ferroelectric ceramics. However, to obtain the ferroelectric properties[75, 76], such organic materials required a poling field 10 times higher than conventional ceramics[77], resulting in dielectric breakdown of material. This disadvantage can be circumvented by performing the terpolymer technology under pseudo-piezoelectric configuration. Indeed, the piezoelectric properties of the terpolymer were equivalent to those of the ferroelectric polymer for an electric field of $7.5 \mathrm{~V} / \mu \mathrm{m}$, which was 8 times lower than the conventional polarization electric field of the copolymer $\mathrm{P}(\mathrm{VDF}-\mathrm{TrFE})$. On the other hand, both terpolymer and copolymer exhibited a limited temperature range as opposed to the classical ceramics. Table 2 summarized 
the key parameters of each technologies. In conclusion, the main advantage of the terpolymer involved in its excellent electromechanical properties, making it possible to operating such a material in both actuator (high strain response) and sensor modes (sensitivity equivalent to the ferroelectric polymer). This opens a new access to a great freedom of design in terms of transducer integration, e.g. smart guidewires in vascular surgery, haptic interface device with force feedback, control of deformable mirror and adaptive optics for astronomy application[78], and so on. This freedom is even more important thanks to the process of inkjet printing technique where the complex device structures as well as multilayer layout can be readily fabricated.

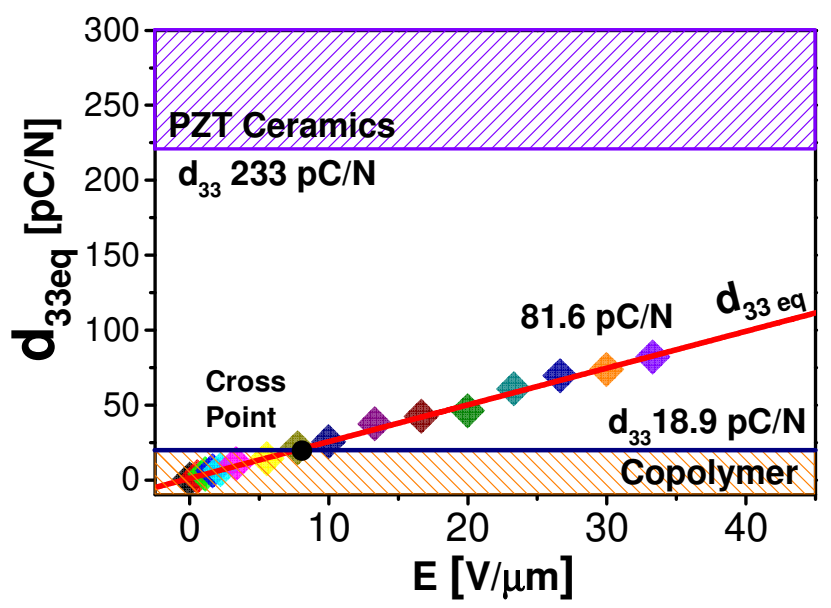

Figure 17 Terpolymer pseudo-piezoelectric coefficient $d_{33 e q}$ as a function of the bias electric field $E$. The red line is the linear fit to the pseudo-piezoelectric coefficient $d_{33 e q}$ against $E$ and the horizontal solid line is assigned to the electrically independent piezoelectric coefficient $d_{33}$ of the printed copolymer sensors and extensively studied PZT ceramics.

Table 2 A comparison of electromechanical properties for the both printed force sensors in this present work and most investigated PZT ceramics.

\begin{tabular}{|c|c|c|c|}
\hline Acronyms & Printed Copolymer & Printed Terpolymer & PZT Ceramics \\
\hline Young’s modulus @ R.T. & a3.3 GPa & $182 \mathrm{MPa}$ & ${ }^{\mathrm{b}} 60 \mathrm{GPa}$ \\
\hline Density $\left(\mathrm{g} \mathrm{cm}^{-3}\right)$ & 1.78 [79] & $\sim 1.3[80]$ & $\sim 7.80[81]$ \\
\hline Dielectric Permittivity & $10.8(1 \mathrm{~K} \mathrm{~Hz})$ & $44.5(1 \mathrm{~K} \mathrm{~Hz})$ & 1200 \\
\hline Dielectric Strength $\left(\mathrm{V} \mu \mathrm{m}^{-1}\right)$ & $\leq 80$ [82] & $269[20]$ & $64[83]$ \\
\hline$P_{r}\left(\mathrm{mC} \mathrm{m}^{-2}\right)$ & $65 \mathrm{mC} \mathrm{m}^{-2}$ & -- & -- \\
\hline$P_{s}$ at $70 \mathrm{~V} / \mu m\left(\mathrm{mC} \mathrm{m}^{-2}\right)$ & $72 \mathrm{mC} \mathrm{m}^{-2}$ & -- & -- \\
\hline 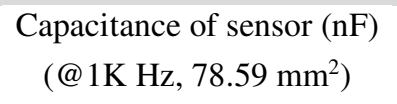 & 0.68 & 3.08 & -- \\
\hline $\begin{array}{l}\text { Piezoelectric coefficient } \\
\qquad\left(\mathrm{pC} \mathrm{N}^{-1}\right)\end{array}$ & $\sim 18.9$ & $\begin{array}{c}18.9 @ 7.5 \mathrm{~V} / \mu \mathrm{m} \\
81.6 @ 33.33 \mathrm{~V} / \mu \mathrm{m}\end{array}$ & 223 [84] \\
\hline${ }^{\circ}$ Powder Cost $\left(€ \mathrm{~g}^{-1}\right)$ & 7 & 9 & 15.2 \\
\hline
\end{tabular}

Note: a, value provided by reference [14]; $b$, value provided by reference [84]; $c$, refers to the Powder indicative Price List [85] 


\section{Conclusions}

Inkjet printed organic electronics have great potential when it comes to applications demanding low cost/power consumption, large areas, flexibility and miniaturization. As one of the most industrially used electronic devices, miniaturized force sensors attract a huge amount of attention due to the possibility of using them in tactile sensing elements of robotics and wearable equipment. In the present work, we demonstrated an inkjet printed sensor based on an electrostrictive terpolymer exhibiting the best electromechanical coupling effect to date. The ink-jettable parameters were first adapted to the physical nature of the polymer ink solution. The inkjet printing protocol for the ferroelectric copolymer and electrostrictive terpolymer was subsequently optimized.

Most importantly for a copolymer sensor, it did not work properly as a force-sensing device unless it was poled at a high electric field. The remnant polarization $P_{r}$ was as high as $65 \mathrm{mC} \mathrm{m} \mathrm{m}^{-2}$ compared with the inkjet printed sensor devices based on the electrostrictive terpolymer, where the latter failed to measure the $P_{r}$. An electric field bias is compulsory in order to induce the polarization, which was not the case for the copolymer sensor. The performance of both printed electroactive polymer sensors was carefully investigated by applying a dynamic force using an impact hammer. Consequently, the copolymer sensor had a typical piezoelectric constant of $18.7 \mathrm{pC} \mathrm{N}^{-1}$ coinciding with the best results reported in the literature. The printed terpolymer sensor, on the other hand, showed a dependence of the piezoelectric constant on the bias electric field. In comparison, it was remarkable that for a given electromechanical performance the bias electric field for the printed electrostrictive terpolymer was much lower than the corresponding poling electric field for a printed copolymer sensor. It was also observed that piezoelectric properties equivalent to those of a corresponding printed copolymer sensor could be obtained for a bias as low as $7.5 \mathrm{~V} / \mu \mathrm{m}$.

These results reliably demonstrate the feasibility of electromechanical sensor devices based on a lowcost inkjet printed electrostrictive terpolymer with performances comparable to printed ferroelectric copolymer sensors at the expense of only a fairly low bias electric field. These findings are rather promising for the moment, although future work should focus on all printed and multi-stack structured electronic devices with the aim of enhancing the mechanical energy density.

\section{Acknowledgement}

The research leading to these results was funded by joint project "Development of Fully-printed Fluorinated Copolymers for Pyroelectric IR sensors". The authors thank Dr. Florent Ganet for his advice on inkjet printing of electroactive polymer layers and device fabrication and Mr. Laurent Lebrun for the fruitful discussions and guidance on data analysis. Finally, Dr. Qing Liu gratefully acknowledges the Chinese Scholarship Council (CSC) for a doctoral fellowship.

\section{Notes}

The authors declare no competing financial interest. 


\section{References}

1. Schwizer, J., M. Mayer, and O. Brand, Force sensors for microelectronic packaging applications. 2006: Springer Science \& Business Media.

2. Kim, K., et al., Nanonewton force-controlled manipulation of biological cells using a monolithic MEMS microgripper with two-axis force feedback. Journal of Micromechanics and Microengineering, 2008. 18(5): p. 055013.

3. $\quad$ Crocker, M.J., Handbook of acoustics. 1998: John Wiley \& Sons.

4. Merril, G.L., et al., Apparatus for controlling force for manipulation of medical instruments. 16 Nov. 2004, U.S. Patent

5. Subramanian, V., et al. Printed electronics for low-cost electronic systems: Technology status and application development. in Solid-State Circuits Conference, 2008. ESSCIRC 2008. 34th European. 2008. IEEE.

6. Khan, S., L. Lorenzelli, and R.S. Dahiya, Technologies for printing sensors and electronics over large flexible substrates: a review. IEEE Sensors Journal, 2015. 15(6): p. 3164-3185.

7. Teichler, A., J. Perelaer, and U.S. Schubert, Inkjet printing of organic electronics-comparison of deposition techniques and state-of-the-art developments. Journal of Materials Chemistry C, 2013. 1(10): p. 1910-1925.

8. Singh, M., et al., Inkjet printing - process and its applications. Advanced materials, 2010. 22(6): p. 673-685.

9. de Gans, B.J., P.C. Duineveld, and U.S. Schubert, Inkjet printing of polymers: state of the art and future developments. Advanced materials, 2004. 16(3): p. 203-213.

10. De Gans, B.-J. and U.S. Schubert, Inkjet printing of well-defined polymer dots and arrays. Langmuir, 2004. 20(18): p. 7789-7793.

11. DeGraff, J., et al., Printable low-cost and flexible carbon nanotube buckypaper motion sensors. Materials \& Design, 2017. 133: p. 47-53.

12. Le, M.Q., et al., Printing of microstructure strain sensor for structural health monitoring. Applied Physics A, 2017. 123(5): p. 354.

13. Zhang, Q., V. Bharti, and X. Zhao, Giant electrostriction and relaxor ferroelectric behavior in electron-irradiated poly (vinylidene fluoride-trifluoroethylene) copolymer. Science, 1998. 280(5372): p. 2101-2104.

14. Xia, F., et al., High electromechanical responses in a poly (vinylidene fluoride-trifluoroethylene-chlorofluoroethylene) terpolymer. Advanced Materials, 2002. 14(21): p. 1574-1577.

15. Zhang, Q., et al., An all-organic composite actuator material with a high dielectric constant. Nature, 2002. 419(6904): $p$. 284-287.

16. Huang, C. and Q. Zhang, Enhanced Dielectric and Electromechanical Responses in High Dielectric Constant All - Polymer Percolative Composites. Advanced Functional Materials, 2004. 14(5): p. 501-506.

17. Yin, X., J.-F. Capsal, and D. Guyomar, A comprehensive investigation of poly (vinylidene fluoride-trifluoroethylenechlorofluoroethylene) terpolymer nanocomposites with carbon black for electrostrictive applications. Applied Physics Letters, 2014. 104(5): p. 052913.

18. Xie, Y., et al., Fabrication of Stretchable Nanocomposites with High Energy Density and Low Loss from Crosslinked PVDF Filled with Poly (dopamine) Encapsulated BaTiO3. ACS Applied Materials \& Interfaces, 2017.

19. Bhavanasi, V., D.Y. Kusuma, and P.S. Lee, Polarization Orientation, Piezoelectricity, and Energy Harvesting Performance of Ferroelectric PVDF - TrFE Nanotubes Synthesized by Nanoconfinement. Advanced Energy Materials, 2014. 4(16).

20. Yin, X., et al., Enhanced Electromechanical Performances in Plasticizer Modified Electrostrictive Polymers. European Polymer Journal, 2016.

21. Liu, Q., C. Richard, and J.-F. Capsal, Control of crystal morphology and its effect on electromechanical performances of electrostrictive P(VDF-TrFE-CTFE) terpolymer. European Polymer Journal, 2017. 91: p. 46-60.

22. IEEE Standard on Piezoelectricity. , in ANSI/IEEE Std 1988. p. 176-1987.

23. Kawai, H., The piezoelectricity of poly (vinylidene fluoride). Japanese Journal of Applied Physics, 1969. 8(7): p. 975.

24. Liu, Y., et al., Investigation of electrostrictive polymers for energy harvesting. ieee transactions on ultrasonics, ferroelectrics, and frequency control, 2005. 52(12): p. 2411-2417.

25. Ren, K., et al., An active energy harvesting scheme with an electroactive polymer. Applied physics letters, 2007. 91(13): p. 132910.

26. Cottinet, P.-J., et al., Investigation of electrostrictive polymer efficiency for mechanical energy harvesting. IEEE transactions on ultrasonics, ferroelectrics, and frequency control, 2011. 58(9): p. 1842-1851.

27. Cottinet, P.-J., et al., Analysis of AC-DC conversion for energy harvesting using an electrostrictive polymer P (VDF-TrFECFE). IEEE transactions on ultrasonics, ferroelectrics, and frequency control, 2011. 58(1): p. 30-42.

28. Guyomar, D., et al., Electrostrictive energy conversion in polyurethane nanocomposites. Journal of Applied Physics, 2009. 106(1): p. 014910.

29. Yin, X., et al., Mechanical energy harvesting via a plasticizer-modified electrostrictive polymer. Applied Physics Letters, 2016. 108(4): p. 042901.

30. Ganet, F., et al., Haptic feedback using an all-organic electroactive polymer composite. Sensors and Actuators B: Chemical, 2015. 220: p. 1120-1130. 
31. Piezotech, https://www.piezotech.eu/en/Technical-center/Documentation/.

32. de Gans, B.J., et al., Ink - jet Printing Polymers and Polymer Libraries Using Micropipettes. Macromolecular Rapid Communications, 2004. 25(1): p. 292-296.

33. Li, J. and F. Yan, Solution-Processable Low-Voltage and Flexible Floating-Gate Memories Based on an n-Type Polymer Semiconductor and High-k Polymer Gate Dielectrics. ACS applied materials \& interfaces, 2014. 6(15): p. 12815-12820.

34. He, F., et al., Preparation and characterization of porous poly (vinylidene fluoride-trifluoroethylene) copolymer membranes via electrospinning and further hot pressing. Polymer Testing, 2011. 30(4): p. 436-441.

35. Chen, Y., et al., Solution - Processed Highly Efficient Alternating Current - Driven Field - Induced Polymer Electroluminescent Devices Employing High - $k$ Relaxor Ferroelectric Polymer Dielectric. Advanced Functional Materials, 2014. 24(11): p. 1501-1508.

36. Yeow, M., Y. Liu, and K. Li, Morphological study of poly (vinylidene fluoride) asymmetric membranes: effects of the solvent, additive, and dope temperature. Journal of Applied Polymer Science, 2004. 92(3): p. 1782-1789.

37. 16, A.D.-. Standard Test Method for Apparent Viscosity of Plastisols and Organosols at Low Shear Rates. 1995.

38. Somani, S., E.S. Shaqfeh, and J.R. Prakash, Effect of Solvent Quality on the Coil- Stretch Transition. Macromolecules, 2010. 43(24): p. 10679-10691.

39. Dong, H., W.W. Carr, and J.F. Morris, An experimental study of drop-on-demand drop formation. Physics of Fluids (1994present), 2006. 18(7): p. 072102.

40. Fromm, J., Numerical calculation of the fluid dynamics of drop-on-demand jets. IBM Journal of Research and Development, 1984. 28(3): p. 322-333.

41. Jang, D., D. Kim, and J. Moon, Influence of fluid physical properties on ink-jet printability. Langmuir, 2009. 25(5): p. 26292635.

42. Migler, K.B., String formation in sheared polymer blends: Coalescence, breakup, and finite size effects. Physical review letters, 2001. 86(6): p. 1023.

43. Derby, B., Inkjet printing of functional and structural materials: fluid property requirements, feature stability, and resolution. Annual Review of Materials Research, 2010. 40: p. 395-414.

44. Mcllroy, C., O. Harlen, and N. Morrison, Modelling the jetting of dilute polymer solutions in drop-on-demand inkjet printing. Journal of Non-Newtonian Fluid Mechanics, 2013. 201: p. 17-28.

45. Smith, D.E., H.P. Babcock, and S. Chu, Single-polymer dynamics in steady shear flow. Science, 1999. 283(5408): p. 17241727.

46. Haskal, E.I., et al., 21.1: Ink Jet Printing of Passive-Matrix Polymer Light Emitting Displays. SID Symposium Digest of Technical Papers, 2002. 33(1): p. 776-779.

47. Rubaiyet Iftekharul, H., et al., Inkjet printing of high molecular weight PVDF-TrFE for flexible electronics. Flexible and Printed Electronics, 2016. 1(1): p. 015001.

48. Christanti, Y. and L.M. Walker, Surface tension driven jet break up of strain-hardening polymer solutions. Journal of NonNewtonian Fluid Mechanics, 2001. 100(1): p. 9-26.

49. Nallan, H.C., et al., Systematic Design of Jettable Nanoparticle-Based Inkjet Inks: Rheology, Acoustics, and Jettability. Langmuir, 2014. 30(44): p. 13470-13477.

50. Tai, J., et al. Control of droplet formation in inkjet printing using Ohnesorge number category: materials and processes. in Electronics Packaging Technology Conference, 2008. EPTC 2008. 10th. 2008. IEEE.

51. Derby, B. and N. Reis, Inkjet printing of highly loaded particulate suspensions. Mrs Bulletin, 2003. 28(11): p. 815-818.

52. Meixner, R.M., et al., Characterization of polymer inks for drop-on-demand printing systems. Microsystem technologies, 2008. 14(8): p. 1137-1142.

53. Tai, Y.-L. and Z.-G. Yang, Flexible, Transparent, Thickness-Controllable SWCNT/PEDOT: PSS Hybrid Films Based on CoffeeRing Lithography for Functional Noncontact Sensing Device. Langmuir, 2015. 31(48): p. 13257-13264.

54. Wong, T.-S., et al., Nanochromatography driven by the coffee ring effect. Analytical chemistry, 2011. 83(6): p. 18711873.

55. Choi, S., et al., Coffee-ring effect-based three dimensional patterning of micro/nanoparticle assembly with a single droplet. Langmuir, 2010. 26(14): p. 11690-11698.

56. Zhang, Z., et al., Controlled inkjetting of a conductive pattern of silver nanoparticles based on the coffee - ring effect. Advanced Materials, 2013. 25(46): p. 6714-6718.

57. Tekin, E., et al., Inkjet printing of luminescent CdTe nanocrystal-polymer composites. Advanced Functional Materials, 2007. 17(1): p. 23-28.

58. Rudawska, A. and E. Jacniacka, Analysis for determining surface free energy uncertainty by the Owen-Wendt method. International Journal of Adhesion and Adhesives, 2009. 29(4): p. 451-457.

59. Still, T., P.J. Yunker, and A.G. Yodh, Surfactant-induced Marangoni eddies alter the coffee-rings of evaporating colloidal drops. Langmuir, 2012. 28(11): p. 4984-4988.

60. Dua, V., et al., All - organic vapor sensor using inkjet - printed reduced graphene oxide. Angewandte Chemie International Edition, 2010. 49(12): p. 2154-2157. 
61. Valeton, J.J., et al., Room temperature preparation of conductive silver features using spin-coating and inkjet printing. Journal of Materials Chemistry, 2010. 20(3): p. 543-546.

62. Lim, J.A., et al., Self - Organization of Ink - jet - Printed Triisopropy/silylethynyl Pentacene via Evaporation - Induced Flows in a Drying Droplet. Advanced functional materials, 2008. 18(2): p. 229-234.

63. Park, J. and J. Moon, Control of colloidal particle deposit patterns within picoliter droplets ejected by ink-jet printing. Langmuir, 2006. 22(8): p. 3506-3513.

64. Robin, M., et al., Epoxy based ink as versatile material for inkjet-printed devices. ACS applied materials \& interfaces, 2015. 7(39): p. 21975-21984.

65. Kim, D., et al., Direct writing of silver conductive patterns: Improvement of film morphology and conductance by controlling solvent compositions. Applied Physics Letters, 2006. 89(26): p. 264101.

66. Wang, L., et al., Inkjet printed colloidal photonic crystal microdot with fast response induced by hydrophobic transition of poly (N-isopropyl acrylamide). Journal of Materials Chemistry, 2012. 22(40): p. 21405-21411.

67. Soltman, D. and V. Subramanian, Inkjet-printed line morphologies and temperature control of the coffee ring effect. Langmuir, 2008. 24(5): p. 2224-2231.

68. Saito, Y., et al., Lead-free piezoceramics. Nature, 2004. 432(7013): p. 84.

69. Tan, Y., et al., Unfolding grain size effects in barium titanate ferroelectric ceramics. Scientific reports, 2015. 5.

70. Zirkl, M., et al., An All - Printed Ferroelectric Active Matrix Sensor Network Based on Only Five Functional Materials Forming a Touchless Control Interface. Advanced Materials, 2011. 23(18): p. 2069-2074.

71. Khan, S., et al., Flexible tactile sensors using screen-printed P (VDF-TrFE) and MWCNT/PDMS composites. IEEE Sensors Journal, 2015. 15(6): p. 3146-3155.

72. Oliveira, F., et al., Process influences on the structure, piezoelectric, and gas - barrier properties of PVDF - TrFE copolymer. Journal of Polymer Science Part B: Polymer Physics, 2014. 52(7): p. 496-506.

73. Ganet, F., et al., Development of a smart guide wire using an electrostrictive polymer: option for steerable orientation and force feedback. Scientific Reports, 2015. 5: p. 18593.

74. Le, M.Q., et al., All-organic electrostrictive polymer composites with low driving electrical voltages for micro-fluidic pump applications. Scientific Reports, 2015. 5: p. 11814.

75. Giacometti, J.A., G.F.L. Ferreira, and B. Gross. A summary of corona charging methods. in 6th International Symposium on Electrets,(ISE 6) Proceedings. 1988.

76. Tasaka, S., M. Kawaguchi, and N. Inagaki, Ferroelectric behavior in poly(6-propiolactone). European Polymer Journal, 1998. 34(12): p. 1743-1745.

77. Perls, T.A., T.J. Diesel, and W.I. Dobrov, Primary Pyroelectricity in Barium Titanate Ceramics. Journal of Applied Physics, 1958. 29(9): p. 1297-1302.

78. Kuhn, J.R., et al. The Exo-Life Finder Telescope (ELF): design and beam synthesis concepts. in SPIE Astronomical Telescopes + Instrumentation. 2018. SPIE.

79. Lee, Y.-S., G. Collins, and T.L. Arinzeh, Neurite extension of primary neurons on electrospun piezoelectric scaffolds. Acta biomaterialia, 2011. 7(11): p. 3877-3886.

80. Huang, C. and Q. Zhang. High-dielectric-constant polymers as high-energy-density (HED) field effect actuator and capacitor materials. in Smart Structures and Materials. 2004. International Society for Optics and Photonics.

81. Laurenti, M., et al., Development of a flexible lead-free piezoelectric transducer for health monitoring in the space environment. Micromachines, 2015. 6(11): p. 1729-1744.

82. Dahiya, R.S., et al. Deposition, processing and characterization of P (VDF-TrFE) thin films for sensing applications. in Sensors, 2008 IEEE. 2008. IEEE.

83. Chen, H.D., et al., Fabrication and electrical properties of lead zirconate titanate thick films. Journal of the American Ceramic Society, 1996. 79(8): p. 2189-2192.

84. Muralt, P., PZT thin films for microsensors and actuators: Where do we stand? IEEE transactions on ultrasonics, ferroelectrics, and frequency control, 2000. 47(4): p. 903-915.

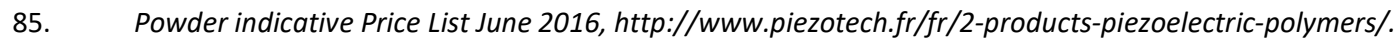




\section{Supporting information}

\section{Enhanced Pseudo-piezoelectric Dynamic Force Sensors based on Inkjet-printed Electrostrictive Terpolymer}

Qing Liu ${ }^{a}$, Minh Quyen Le ${ }^{a}$, Claude Richard ${ }^{a}$, Richard Liang ${ }^{b}$, Pierre-Jean Cottinet ${ }^{a}$, Jean-Fabien Capsal*a

${ }^{a}$ Université de Lyon, INSA-Lyon, LGEF, EA682, F-69621, Villeurbanne, France.

${ }^{b}$ High-Performance Materials Institute, FAMU-FSU College of Engineering, Florida State University 2005 Levy Ave, Tallahassee, FL 32310, United States

*Correspondence to: J.F. Capsal (E-mail: jean-fabien.capsal@insa-lyon.fr)

Table S1 Optimal inkjet printing protocol used for generating a printable droplet formation of copolymer and terpolymer solution inks in this present study

\begin{tabular}{|c|c|c|c|c|c|}
\hline Parameters & Dimension & Copolymer inks & Terpolymer inks & Note & \\
\hline Ink Pressure & mBar & -21.7 & -21.9 & & \\
\hline Number of active nozzles & & 25 & 20 & & \\
\hline \multirow[b]{4}{*}{ Firing frequency } & $\mu s$ & 8 & 8 & & \\
\hline & $\mu s$ & 7 & 6 & & \\
\hline & $\mu s$ & 4 & 4 & & \\
\hline & $\mathrm{Hz}$ & 1000 & 1000 & & \\
\hline Nozzle Temperature & ${ }^{\circ} \mathrm{C}$ & 70 & 70 & & \\
\hline Droplet velocity & $\mathrm{m} \mathrm{s}^{-1}$ & $0.87 \pm 0.05$ & $0.77 \pm 0.02$ & & \\
\hline Droplet size & picoliter & $49.8 \pm 2.1$ & $52.1 \pm 1.8$ & $\begin{array}{l}\text { Referred } \\
\text { Printed }\end{array}$ & to \\
\hline Substrate temperature & ${ }^{\circ} \mathrm{C}$ & 90 & 90 & & \\
\hline Number of printed layers & & 5 & 5 & & \\
\hline Thickness & $\mu m$ & 10 & 11 & measured & \\
\hline Thickness per layer & $\mu m$ & 2 & 2.2 & & \\
\hline Reynolds Re & dimensionless & 2351.2 & 1277.82 & calculated & \\
\hline Weber We & dimensionless & 1514.2 & 960.99 & calculated & \\
\hline Ohnesorge Oh & dimensionless & 0.01655 & 0.02426 & calculated & \\
\hline $\mathrm{Z}=1 / \mathrm{Oh}$ & dimensionless & 61.54 & 42.22 & calculated & \\
\hline
\end{tabular}




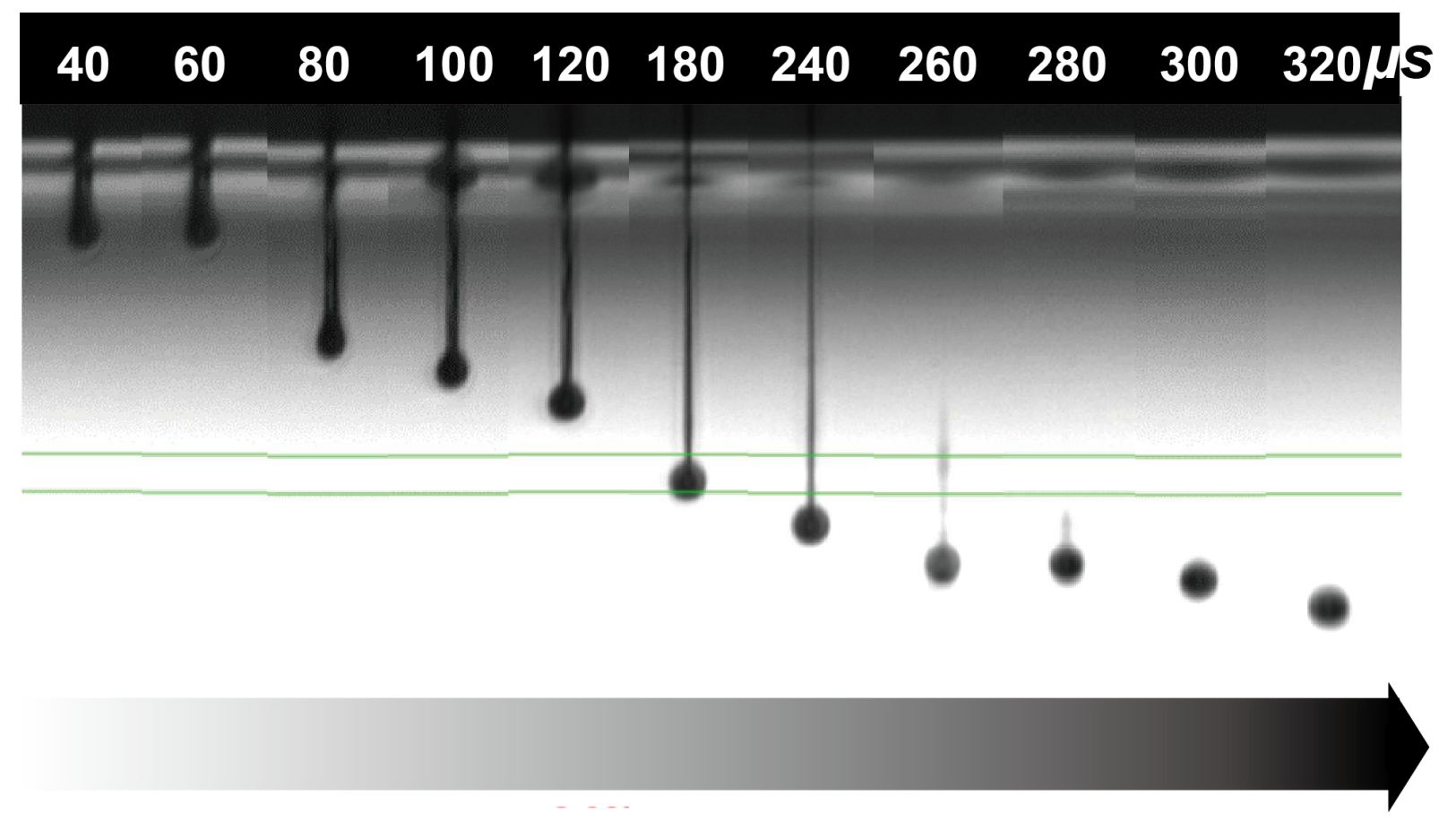

Figure S. 1 Snapshot graphics showing the droplet morphology evolution over time elapsing. $1 \mathrm{wt} \%$ $\mathrm{P}$ (VDF-TrFE-CTFE) in low volatile TEP solvent. The polymer solution droplet detached to the nozzle through inks liquid filament as time elapse beyond $260 \mu \mathrm{s}$. Green horizontal bars marked the same altitude position of the stroboscopic snapshots. 

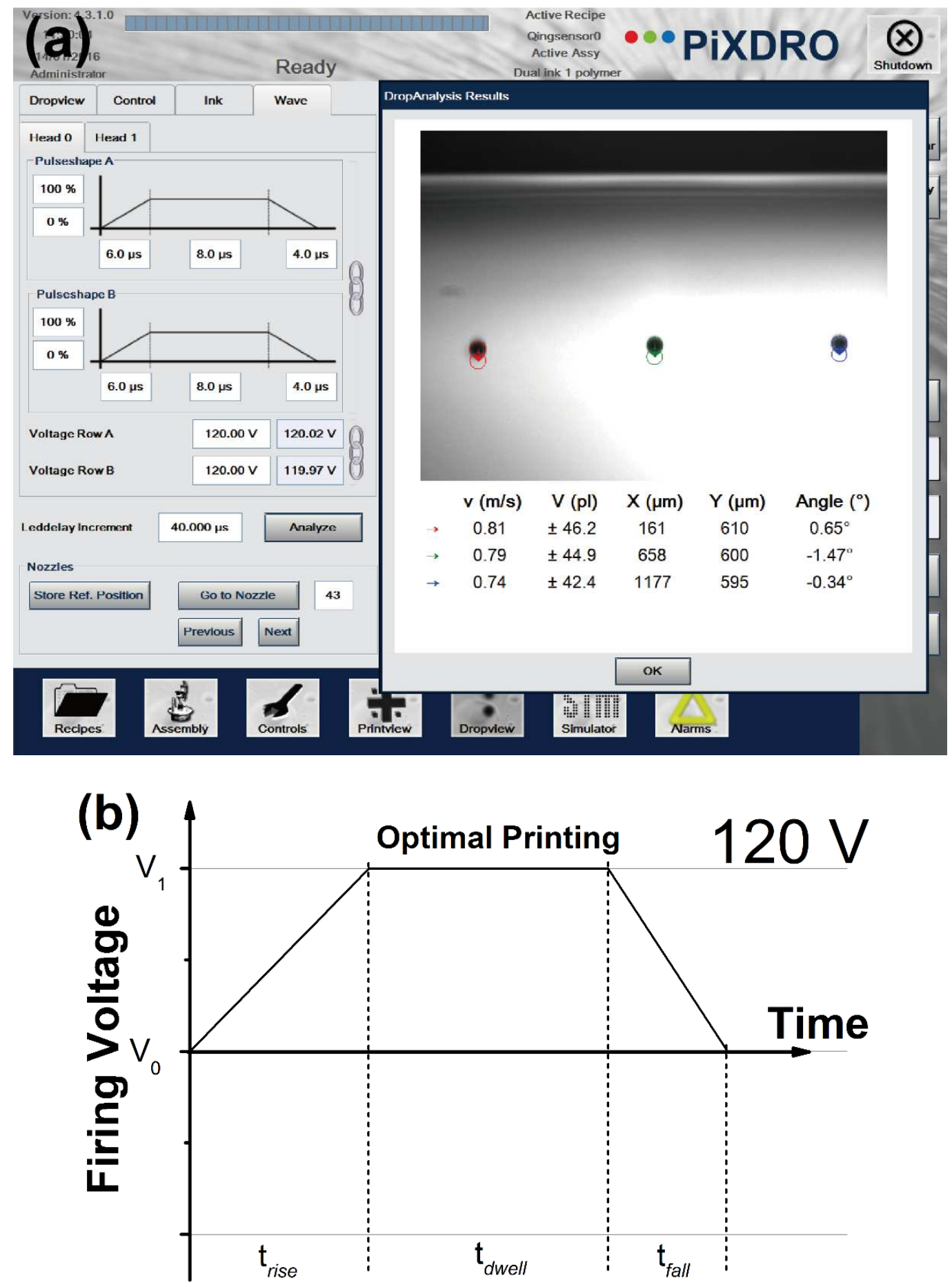

Figure S. 2 (a) Snapshot of a waveform applied on the Pixdro-LP50 inkjet printer while inkjet printing of 1 wt\% terpolymer inks. (b) Optimal diagram of bipolar voltage pulse waveform for inkjet printing. The print head was powered by a voltage of $120 \mathrm{~V}$, with a pulse width of $6 \mu$ s for the raising step $\left(t_{\text {rise }}\right), 8 \mu \mathrm{s}$ for the dwelling step $\left(t_{d w e l l}\right)$ and $4 \mu$ s for the falling step $\left(t_{\text {fall }}\right)$. 
The drop volume (size) and droplet velocity strongly differ from system to system. However, all ink systems have the similar behavior as if the pulse waves applied for the piezoelectric nozzle are kept unaltered. The droplets ejection intend to occur during the positive trapezoidal pulse $\left(t_{d w e l l}\right)$, as shown in Figure S. 2. The droplet size could be determined by the time duration of the steady wave stage. The longer $t_{d w e l l}$, the larger the droplet size. And the rising ramp time span and falling ramp time spans were thought to be as short as possible because the longer ramp times would disturb the droplet formation and thus yield undesirable and inhomogeneous drop volume. Negative wave pulse $\left(t_{\text {echo }}\right)$ was set in order to counteract the residual deformation waves whereby unwanted excessive liquid flows were sustained in the orifice right after the droplet ejection which was set to 0 second in our inkjet printing protocol.

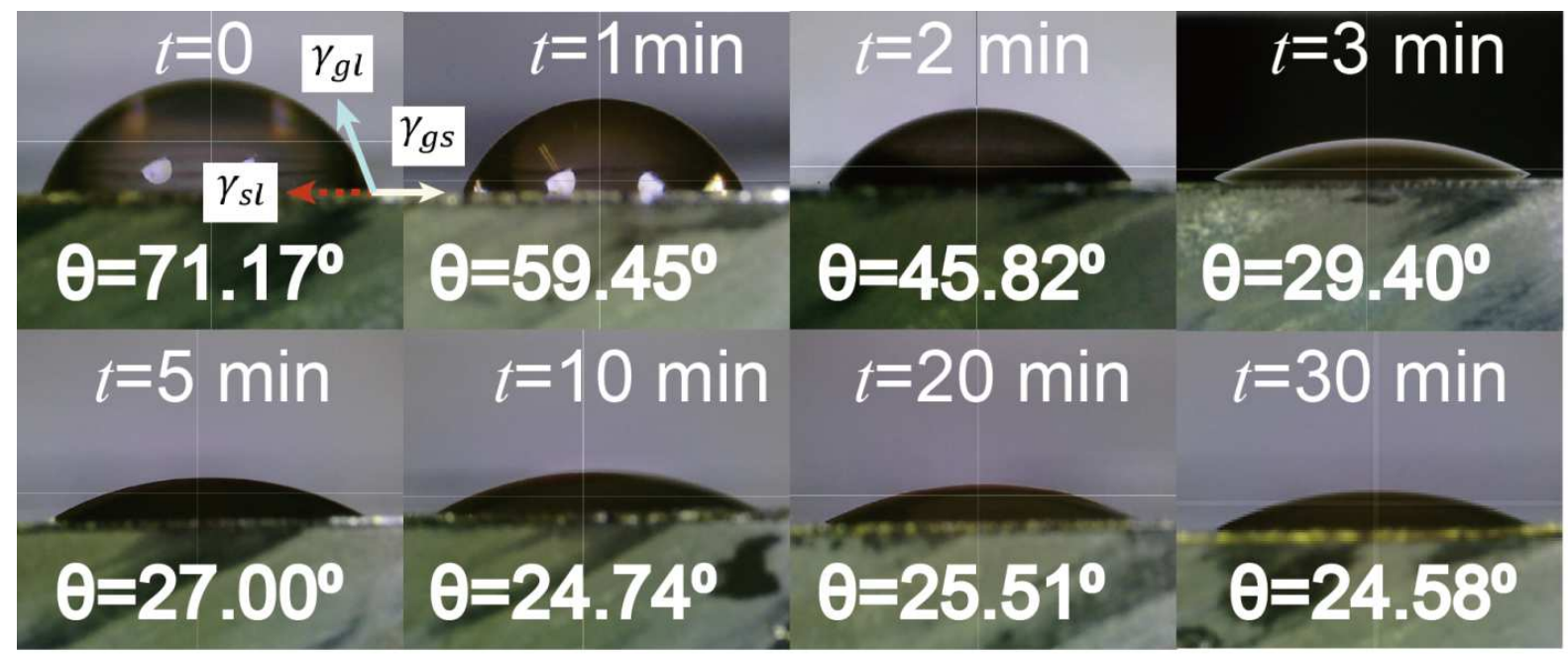

Figure S. 3 Photographics of the water droplets on the pristine PI against UV-Ozone treatment times. $\gamma_{g l}$ represents the water surface tension vector; $\gamma_{s g}$ symbolizes the PI foil surface tension veactor; $\gamma_{s l}$ denotes the surface tension of PI foil and water interface. $\theta$ is the contact angle of the water drops on the untreated and treated PI foil substrate. 

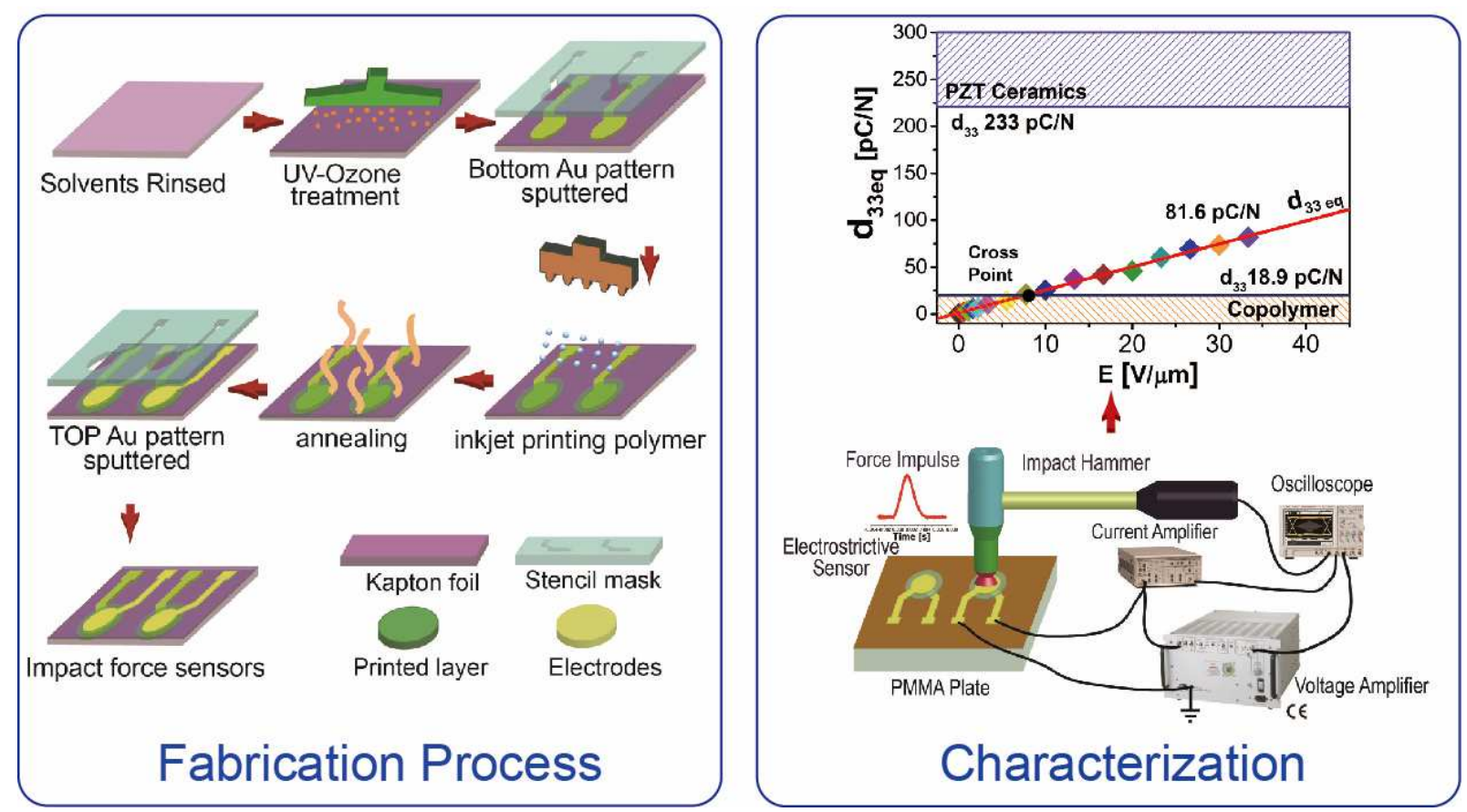

\section{Graphical Abstract}

